\title{
Chemical composition of Galactic OB stars
}

\section{CNO abundances in 09 stars $^{\star}$}

\author{
M. R. Villamariz ${ }^{1}$, A. Herrero ${ }^{1,2}$, S. R. Becker ${ }^{3}$, and K. Butler ${ }^{3}$ \\ 1 Instituto de Astrofísica de Canarias, 38200 La Laguna, Tenerife, Spain \\ 2 Departamento de Astrofísica, Universidad de La Laguna, Avda. Astrofísico Francisco Sánchez, s/n, \\ 38071 La Laguna, Spain \\ 3 Universitäts-Sternwarte München, Scheinerstr. 1, 81679 München, Germany
}

Received 20 November 2001 / Accepted 8 March 2002

\begin{abstract}
We present NLTE abundances of CNO for a sample of four O9 stars in the Galaxy, together with new determinations of their stellar parameters, $T_{\text {eff }}, \log g, \epsilon(\mathrm{He})$ and microturbulence. These new analyses take into account the effect of line-blocking in the spectral synthesis with our classical NLTE, plane-parallel and hydrostatic model atmospheres. The sample includes three O9 He normal stars: two dwarfs, HD 214680 and HD 34078, and one supergiant, HD 209975, and one fast rotating giant with a preliminary high He overabundance, HD 191423 with $\epsilon(\mathrm{He})=0.20$. We find first that the consideration of microturbulence in the spectral synthesis for the fast rotator leads to a considerably lower He abundance, $\epsilon(\mathrm{He})=0.12$. The $\mathrm{CNO}$ abundances of the three He normal stars are in good agreement with the values in the literature for Galactic B dwarfs with no evidence of mixing, and show that they all have the same chemical composition. We also discuss however the possible CNO contamination of the supergiant HD 209975. For the fast rotator we find that the abundances show the trend of the CNO contamination: a $\mathrm{N}$ overabundance together with $\mathrm{C}$ and $\mathrm{O}$ depletion. The $\mathrm{N} / \mathrm{C}$ and $\mathrm{N} / \mathrm{O}$ ratios of our stars as a function of their projected rotational velocities are consistent with the predictions of the recent evolutionary models of Meynet \& Maeder (2000).
\end{abstract}

Key words. stars: atmospheres - stars: early-type - stars: fundamental parameters - stars: abundances stars: evolution

\section{Introduction}

Massive blue stars strongly influence the chemical evolution of galaxies by their interaction with the Interstellar Medium, returning to it the material from their atmospheres and interiors. In recent years, there is growing evidence that their atmospheres might be enriched with CNO processed material even in the early stages of their lives, contrary in general to the predictions made by the classical models of stellar evolution (Schaller et al. 1992; Chiosi 1998).

Such models predict that only very massive stars, with masses higher than $60 M_{\odot}$ on the ZAMS, lose enough mass in the wind to show, in the main sequence phase, the inner CNO enriched material (Schaller et al. 1992; Heger 1998).

For lower mass stars, the appearance of CNO processed material in the stellar atmosphere only occurs in later

Send offprint requests to: M. R. Villamariz, e-mail: ccid@ll.iac.es

* The INT is operated on the island of La Palma by the RGO in the Spanish Obervatorio de El Roque de los Muchachos of the Instituto de Astrofísica de Canarias. phases of the evolution, after the first dredge-up (Chiosi 1998).

However, recent investigations on the effect of stellar rotation on the structure and evolution of massive stars (Maeder \& Meynet 2000; Heger 1998; Heger \& Langer 2000) show that early atmospheric contamination can occur by mixing with the stellar interior, this contamination being more important as both the stellar mass and the initial rotational velocity increase.

The physical mechanism responsible for the mixing processes in massive stars may be other than rotation, but rotation appears as a natural driver for this mixing or, at least, as one of the first mechanisms whose consequences on mixing have to be explored (Meynet \& Maeder 2000), especially considering the fact that massive stars are in general quite fast rotators.

Rotationally induced mixing has very important consequences on the evolution of the star, changing the evolutionary tracks towards higher luminosities, widening the main sequence in the HR diagram and making it last longer, and also changing the surface composition of the star. The effects of the $\mathrm{CNO}$ cycle show up in the 
atmosphere, with $\mathrm{He}$ and $\mathrm{N}$ enrichments and $\mathrm{C}$ and $\mathrm{O}$ depletions (Heger \& Langer 2000; Meynet \& Maeder 2000). As Heger \& Langer (2000) show in their Fig. 9, He enrichments appear later in the stellar atmosphere, while CNO abundance changes are already present.

There is plenty of evidence in the literature of this CNO contamination in early type stars, one of the most important being the so-called He discrepancy (Herrero et al. 1992). They find that a considerable fraction of Galactic OB stars are overabundant in He.

With regard to CNO abundances, a wealth of work also finds the trend in these elements abundances produced by the CNO cycle. Gies \& Lambert (1992) find N (and $\mathrm{He}$ ) overabundances in a sample of B supergiants in the Galaxy, while Venn (1995) finds such trend in the abundances of CNO for A supergiants, but not in the $\mathrm{He}$ abundances, that she assumes to have solar values. Other more qualitative works, studying equivalent widths, also find this trend in the $\mathrm{CNO}$ line intensities of B supergiant stars (Lennon et al. 1993; McErlean et al. 1999), but not in the He abundance.

Therefore, no clear correlation between the He overabundance and CNO contamination is found in the literature, maybe due to the fact that, as predicted by rotating evolutionary models, changes in the $\mathrm{N} / \mathrm{C}$ and $\mathrm{N} / \mathrm{O}$ ratios can appear before any He enrichment.

As can be seen from the references given, little work has been done on the calculation of CNO abundances of O stars. Only Schönberner et al. (1988) have studied three stars from O8 to O9 and Pauldrach et al. (1994) and Taresch et al. (1997) two individual Galactic early O stars, $\zeta$ Pup (O4 If) and HD 93129A (O3 If*) respectively.

In the very numerous works on abundances of Galactic B stars (Kilian et al. 1994; Vrancken et al. 2000; Smartt et al. 1997b; Rolleston et al. 2000; Cunha \& Lambert 1994; Daflon et al. 1999; Gummersbach et al. 1998) there is only a marginal presence of late $\mathrm{O}$ stars in the samples studied. Here is where we make our contribution, studying the $\mathrm{CNO}$ abundances of $\mathrm{O}$ type stars in order to compare them with the He abundance.

Of course, abundances derived from spectral analysis are dependent on the model assumptions. For example, as demonstrated by Villamariz (2001) the He abundances obtained for OB stars from NLTE plane-parallel and hydrostatic model atmospheres are lowered by $\sim 20 \%$ (in the $\mathrm{He}$ abundance by number relative to the total $\mathrm{H}+\mathrm{He}, \epsilon(\mathrm{He})$ ) due to the inclusion of line-blocking in the spectral synthesis. A similar effect is found by consideration of a microturbulence of $15 \mathrm{~km} \mathrm{~s}^{-1}$ in the synthesis of the $\mathrm{H}$ and $\mathrm{He}$ lines of supergiant stars (Villamariz \& Herrero 2000), as found earlier for B supergiants by McErlean et al. (1998) and Smith \& Howarth (1998). The combined result is that the He discrepancy is considerably reduced and it even disappears for some stars. However, it still remains for an important fraction of Galactic OB stars.

On the other hand, analyses with the unified code of Santolaya-Rey et al. (1997), which accounts for the stellar wind and considers spherical extension of the atmosphere, show no systematic changes in the He abundance (see Herrero et al. 2000). Indeed, for 2 of the 7 stars analysed in this work, higher He abundances are found (see also Herrero et al. 1995).

In this work we determine the CNO abundances of four 09 stars with known He abundances, as a first step in the investigation of the possible link between rotation and surface $\mathrm{CNO}$ contamination in the whole range of $\mathrm{OB}$ stars. In Sect. 2 we present the objects selected for this study and the equivalent width measurements. In Sect. 3 we present the technical details of our methodology, and in Sect. 4 we make a careful study of the uncertainties in our abundance determinations. Section 5 contains the absolute abundances of the three He normal stars that we analyse, and Sect. 6 their relative values. Section 7 is devoted to the analysis of the fast rotator HD 191423 and finally the discussion and conclussions of our work are given in Sects. 8 and 9 respectively.

\section{The programme stars}

We have studied four O9 stars, two dwarfs and one supergiant with normal He abundances, and one rapidly rotating giant with an important He overabundance $(\epsilon(\mathrm{He})=$ 0.20, Herrero et al. 1992).

Our intention is to make a line by line differential analysis within the four stars, as for example Monteverde et al. (2000) and Smartt et al. (1996, 1997a). The determination of stellar chemical compositions is known to be dependent on the details of the methodology used. That is why it must be done differentially within stars of similar physical conditions. In this way, systematic errors in the line abundances, due to the simplifications adopted in the model atoms for example, can be avoided.

Table 1. The programme stars and their stellar parameters (see text for details). $T_{\text {eff }}$ values are given in thousands of Kelvin, surface gravities in cgs units and the He abundance is the number density of He atoms relative to the total number of $\mathrm{H}+\mathrm{He}$ atoms. Velocities are given in $\mathrm{kms}^{-1}$. The uncertainties in our parameters are the following: $\Delta T_{\text {eff }}=1000 \mathrm{~K}$, $\Delta \log g=0.10$ dex, $\Delta \epsilon(\mathrm{He})=25 \%$ and $\Delta \xi=10 \mathrm{~km} \mathrm{~s}^{-1}$.

\begin{tabular}{cccccc}
\hline \hline Star & $v \sin i$ & $T_{\text {eff }}$ & $\log g$ & $\epsilon(\mathrm{He})$ & $\xi$ \\
\hline HD 214680, O9 V & 50 & 37.5 & 4.00 & 0.10 & 0 \\
HD 34078, O9.5 V & 40 & 36.5 & 4.05 & 0.09 & 0 \\
HD 209975, O9.5 Ib & \multirow{2}{*}{400} & 34.0 & 3.20 & 0.08 & 0 \\
& & 35.0 & 3.25 & 0.06 & 20 \\
HD 191423, O9 III:n & 450 & 34.0 & 3.40 & 0.20 & 0 \\
& & 35.0 & 3.40 & 0.12 & 20 \\
\hline
\end{tabular}

The four stars have been observed by our group during different observing runs. However, the observing conditions, spectral ranges, resolutions and $S / N$ values are very similar in all cases. HD 209975, HD 34078 and HD 191423 were observed with the $2.5 \mathrm{~m}$ Isaac Newton Telescope in 1989 using the IDS spectrograph with the H2400B grating, 
Table 2. Equivalent widths (in $\mathrm{m} \AA$ ) of the $\mathrm{C}$ lines identified in the three stars with normal He abundance (see text for details). Notes for each line in the third column of each star mean: b: maybe blended, di: doubtful identification, or clearly blended and not used, e: excluded from the final abundance determination, n: narrow, with fwhm considerably lower than the rest of the metallic lines. Equivalent widths quoted as - mean that the line is not present or too weak.

\begin{tabular}{|c|c|c|c|c|c|c|c|c|c|c|}
\hline \multirow[t]{2}{*}{ Line } & \multirow{2}{*}{$\begin{array}{l}\text { Multi- } \\
\text { plet }\end{array}$} & \multicolumn{3}{|c|}{ HD 214680} & \multicolumn{3}{|c|}{ HD 34078} & \multicolumn{3}{|c|}{ HD 209975} \\
\hline & & $E W$ & $\Delta E W$ & Notes & $E W$ & $\Delta E W$ & Notes & $E W$ & $\Delta E W$ & Notes \\
\hline C III 4056 & 24 & 34 & 8 & - & - & - & - & - & - & - \\
\hline $\begin{array}{c}\text { C III } 4152 \\
\quad+O \text { II }\end{array}$ & 21 & 121 & 15 & $\mathrm{~b}, \mathrm{e}$ & 137 & 11 & $\mathrm{~b}, \mathrm{e}$ & 40 & 9 & $\mathrm{~b}, \mathrm{e}$ \\
\hline $\begin{array}{c}\text { C III } 4156 \\
+ \text { O II }\end{array}$ & 21 & 64 & 8 & - & 64 & 6 & - & 26 & 9 & - \\
\hline C III 4162 & 21 & 64 & 7 & - & 82 & 8 & - & 34 & 11 & - \\
\hline C III 4186 & 18 & 99 & 7 & - & 122 & 9 & di & 98 & 12 & b, di \\
\hline $\begin{array}{l}\text { C III } 4325 \\
\quad+\mathrm{C}_{\text {II }}+\mathrm{O} \text { II }+\mathrm{N} \text { III }\end{array}$ & 7 & 33 & 6 & - & 51 & 6 & - & 11 & 6 & di, e \\
\hline C III 4647 & 1 & 268 & 9 & - & 267 & 7 & - & - & - & - \\
\hline $\begin{array}{r}\text { C III } 4665 \\
+ \text { SiIII }\end{array}$ & 5 & 55 & 9 & - & 51 & 6 & - & 35 & 9 & - \\
\hline $\begin{array}{c}\text { C III } 4673 \\
+ \text { O II } \\
\end{array}$ & 5 & 41 & 8 & $\mathrm{~b}, \mathrm{e}$ & 40 & 6 & e & - & - & - \\
\hline
\end{tabular}

resulting in a spectral resolution of $0.6 \AA$ (see Herrero et al. 1992), whereas HD 214680 was observed with the same telescope and configuration in a later run in 1992 (see Herrero et al. 2000). The $S / N$ ratios range from 200 to 300 in the $\mathrm{H}_{\beta}$ region.

The stars were analyzed by Herrero et al. (1992) by means of NLTE plane-parallel and hydrostatic $\mathrm{H} / \mathrm{He}$ model atmospheres. Here however we present new parameters with line-blocking included in the line formation calculations, obtained in Villamariz (2001).

Table 1 lists the programme stars together with their parameters. The parameters of HD 209975 and HD 191423 are slightly changed when the microturbulence found from the CNO analysis is applied to the $\mathrm{H} / \mathrm{He}$ analysis, see Sects. 5.3 and 7.1 .

\subsection{Equivalent width measurements}

We have measured equivalent widths of the CNO lines listed in Tables 2 and 3 for the three stars with normal He abundance. The metallic features of the spectrum of the fast rotator HD 191423 (see Sect. 7) are very weak and blended because of the high rotational broadening, and for this star equivalent widths of isolated lines cannot be measured.

When identifying lines in stellar spectra, it is often not easy to know which blends are contributing to the spectrum at any given wavelength. To deal with this difficulty, we have considered all possible contributions to each wavelength listed in the VALD database (Kupka et al. 1999; Ryabchikova et al. 1999) and also in the classical book of Striganov \& Sventitsky (1968), within $\pm 2 \AA$ each side of the line. In Tables 2 and 3 line identifications (in the first column) are given with all possible contributions.

The identification of each spectral line is based on its Doppler shift and on its intensity compared to that of the other members of the multiplet, i.e., we only assume the identification of a line when its Doppler shift is consistent with the rest of the identified lines and its relative intensity compares well to the remaining lines of the multiplet (see Tables 2 and 3 ).

The approximate Gaussian shape of the line gives an idea of the possibility of line blending, and we finally decide about the reliability of each line a posteriori, when all the line abundances for each ion are calculated (see Fig. 1).

To measure the equivalent widths we use our own software developed in IDL. A least squares profile fitting procedure was used, with Gaussian profiles fitting the line and polynomials of degree one or two to fit the local continuum.

Errors in the measurements due to the uncertainty in the position of the local continuum (estimated as $\pm 1 / S / N)$ are quoted together with the equivalent widths. We have found that this is the most important source of uncertainty in the given values, among those coming from the use of Gaussian fits, or from the fitting of the continuum.

We have only considered lines of C III, N III and O II for the abundance determination, because they are the most common ions of each element in our O9 spectra.

\section{Method of analysis}

For the three He normal stars for which equivalent widths of isolated lines are measurable, we make use of the classical method of curve of growth to determine the absolute abundances. For each star and element we proceed as follows. We construct a grid of model spectra for abundances 10 times, 5 times and 2 times higher and lower than solar, and for the solar abundance, each for 6 values of microturbulence: $0,5,10,15,20$ and $25 \mathrm{~km} \mathrm{~s}^{-1}$. 
Table 3. Equivalent widths (in $\mathrm{m} \AA$ ) of the $\mathrm{N}$ and $\mathrm{O}$ lines identified in the three stars with normal He abundance. Notes like in Table 2 .

\begin{tabular}{|c|c|c|c|c|c|c|c|c|c|c|}
\hline \multirow[t]{2}{*}{ Line } & \multirow[t]{2}{*}{ Mult. } & \multicolumn{3}{|c|}{ HD 214680} & \multicolumn{3}{|c|}{ HD 34078} & \multicolumn{3}{|c|}{ HD 209975} \\
\hline & & $E W$ & $\Delta E W$ & Notes & $E W$ & $\Delta E W$ & Notes & $E W$ & $\Delta E W$ & Notes \\
\hline $\begin{array}{l}\mathrm{N} \text { III } 4195 \\
\quad+\mathrm{O} \text { II+N II }\end{array}$ & 6 & 23 & 6 & $\mathrm{e}$ & - & - & - & 25 & 7 & $\mathrm{~b}, \mathrm{e}$ \\
\hline N III 4215 & 6 & 16 & 7 & di, e & - & - & - & 14 & 8 & $\mathrm{e}$ \\
\hline $\begin{array}{l}\text { N III } 4325 \\
\quad+\text { C II-III+O II }\end{array}$ & 10 & 33 & 6 & di, e & 51 & 6 & di, e & 11 & 6 & di, e \\
\hline $\begin{array}{l}\text { N III } 4327 \\
\quad+\mathrm{O} \text { II+SirV }\end{array}$ & 10 & 17 & 9 & di, e & 38 & 7 & b, di, e & 15 & 7 & di, e \\
\hline $\begin{array}{r}\text { N III } 4379 \\
+\mathrm{C}_{\text {III }}\end{array}$ & 17 & 105 & 9 & - & 84 & 6 & di & 132 & 19 & $\mathrm{~b}, \mathrm{e}$ \\
\hline N III 4510 & 3 & 84 & 8 & - & 62 & 6 & - & 136 & 16 & - \\
\hline $\begin{array}{r}\mathrm{N} \text { III } 4514 \\
+\mathrm{C} \text { III }\end{array}$ & 3 & 106 & 7 & - & 109 & 8 & - & 176 & 16 & $\mathrm{~b}$ \\
\hline N III 4523 & 3 & 37 & 7 & - & 34 & 8 & - & 59 & 16 & - \\
\hline N III 4546 & 13 & 17 & 9 & $\mathrm{~b}, \mathrm{e}$ & - & - & - & - & - & - \\
\hline N III 4634 & 2 & 81 & 11 & $\mathrm{e}$ & 60 & 7 & e & - & - & - \\
\hline O II 4072 & 10 & 93 & 9 & - & 92 & 7 & - & - & - & - \\
\hline O Іі 4075 & 10 & 87 & 6 & - & 104 & 6 & - & 78 & 12 & di \\
\hline $\begin{array}{l}\text { O II } 4087 \\
+\mathrm{N} \text { II }\end{array}$ & 48 & 40 & 7 & di & 32 & 6 & $\mathrm{~b}$ & - & - & $\mathrm{b}$ \\
\hline O II 4132 & 19 & 31 & 9 & b & 57 & 10 & $\mathrm{e}$ & 27 & 9 & $\mathrm{e}$ \\
\hline $\begin{array}{r}\text { O II } 4156 \\
\quad+\mathrm{C} \text { III }\end{array}$ & 19 & 64 & 8 & di, e & 65 & 6 & di, e & 29 & 9 & di, e \\
\hline $\begin{array}{r}\text { O II } 4189 \\
+ \text { FeIII }\end{array}$ & 36 & 36 & 7 & - & 58 & 7 & di & - & - & - \\
\hline O II 4253 & 101 & 73 & 10 & $\mathrm{~b}$ & 85 & 7 & - & - & - & - \\
\hline $\begin{array}{r}\text { O II } 4282 \\
+ \text { + } \text { III }\end{array}$ & $67-54$ & 31 & 8 & $\mathrm{~b}, \mathrm{di}$ & - & - & $\mathrm{b}$ & 39 & 12 & $\mathrm{~b}$ \\
\hline $\begin{array}{r}\text { O II } 4288 \\
+\mathrm{N} \text { III }\end{array}$ & 54 & 20 & 5 & di & 28 & 8 & di & - & - & - \\
\hline O II 4294 & 54 & 35 & 9 & - & 39 & 7 & - & 6 & 5 & di, $n$ \\
\hline O II 4303 & 54 & 34 & 6 & - & 61 & 7 & - & 12 & 7 & - \\
\hline $\begin{array}{l}\mathrm{O} \text { II } 4317 \\
\quad+\mathrm{C} \text { II }\end{array}$ & 2 & - & - & - & 89 & 9 & - & - & - & - \\
\hline O II 4319 & 2 & - & - & - & 74 & 7 & - & - & - & - \\
\hline $\begin{array}{l}\text { O II } 4325 \\
\quad+\text { C II-III+N III }\end{array}$ & 2 & 33 & 6 & di, e & 51 & 6 & di, e & 11 & 6 & di, e \\
\hline O II 4349 & 2 & 54 & 6 & - & 75 & 5 & - & 32 & 6 & - \\
\hline $\begin{array}{l}\text { O II } 4366 \\
\quad+\text { C III }\end{array}$ & 2 & 65 & 8 & - & 79 & 7 & - & 72 & 15 & $\mathrm{~b}$ \\
\hline $\begin{array}{l}\text { O II } 4327 \\
\quad+\mathrm{N} \text { III+Sirv }\end{array}$ & 41 & 17 & 9 & di & 38 & 7 & di & 13 & 7 & di \\
\hline $\begin{array}{l}\text { O II } 4369 \\
\quad+\text { C II }\end{array}$ & 26 & 44 & 9 & $\mathrm{~b}, \mathrm{e}$ & 28 & 7 & di & - & - & - \\
\hline $\begin{array}{l}\text { O II } 4414 \\
\quad+\mathrm{C}_{\text {II }}\end{array}$ & $5-60$ & 47 & 9 & - & 67 & 7 & - & 25 & 9 & $\mathrm{~b}$ \\
\hline O II 4596 & 15 & 27 & 6 & - & 40 & 7 & - & 18 & 7 & $\mathrm{n}, \mathrm{e}$ \\
\hline $\begin{array}{l}\text { O II } 4602 \\
\quad+N_{\text {II }}\end{array}$ & 93 & 34 & 9 & $\mathrm{~b}$ & 32 & 7 & $\mathrm{~b}$ & 8 & 6 & $\mathrm{n}$ \\
\hline O II 4610 & $93-92$ & 69 & 12 & $\mathrm{~b}$ & - & - & - & 41 & 14 & $\mathrm{~b}$ \\
\hline $\begin{array}{r}\text { O II } 4638 \\
\text { +SiIII }\end{array}$ & 1 & 66 & 8 & - & 90 & 9 & - & 19 & 6 & di \\
\hline $\begin{array}{r}\text { O II } 4661 \\
+ \text { CuII }\end{array}$ & 1 & 53 & 8 & - & 63 & 5 & - & 24 & 8 & di \\
\hline O II 4676 & 1 & 46 & 7 & - & 62 & 6 & - & - & - & - \\
\hline $\begin{array}{l}\text { O II } 4699 \\
\quad+\mathrm{N}_{\text {II }}\end{array}$ & $40-25$ & 40 & 6 & - & 63 & 6 & - & - & - & - \\
\hline O II 4705 & 25 & 46 & 8 & - & 51 & 5 & - & 16 & 8 & - \\
\hline O II 4943 & 33 & 21 & 5 & - & 40 & 5 & - & - & - & - \\
\hline
\end{tabular}


In this way, we construct the curves of growth for each line that gives the line abundance for each value of microturbulence. With the abundances of all the lines identified in the stellar spectrum we obtain the preliminary absolute abundance of the element together with its particular value of microturbulence (see Sect. 3.3). This value minimizes the dependence of the line abundances with the line strength in the abundance-equivalent width diagrams. These diagrams are also a diagnostic tool to check the reliability of the observed lines for the abundance determination (see Fig. 1).
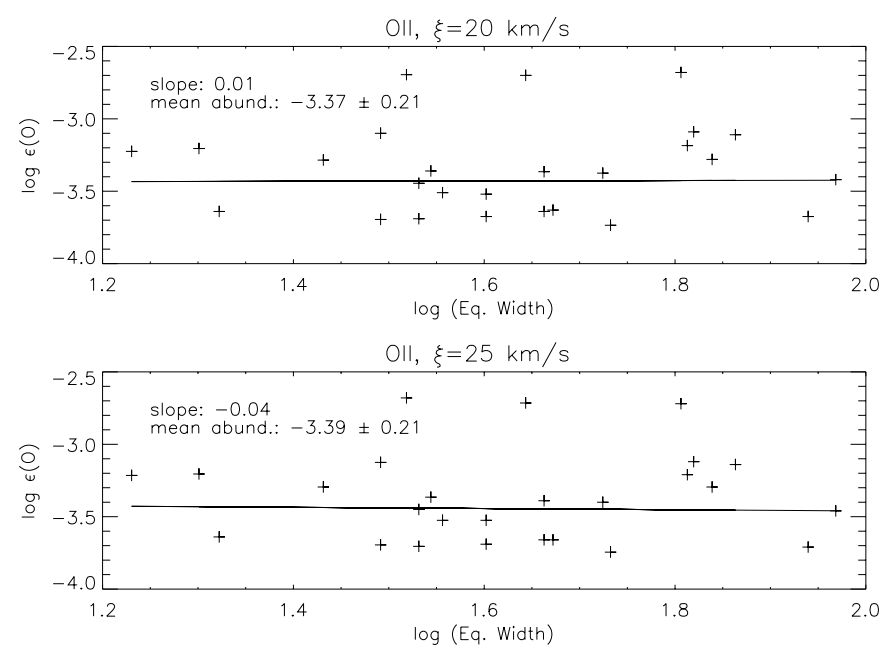

Fig. 1. Abundance vs. equivalent width for all the O II lines found in the spectrum of HD 214680. For a microturbulence between 20 and $25 \mathrm{~km} \mathrm{~s}^{-1}$ the slope reaches 0 . Note how three of the lines (O II 4156, 4325 and 4369) indicate abundances too scattered to be considered.

The model CNO line formation calculations make use of the model atmosphere and model populations of $\mathrm{H}$ and He given by the stellar parameters $T_{\text {eff }}, \log g$ and $\epsilon(\mathrm{He})$ of each star (see Table 2). They are held fixed while the new spectrum is being calculated, with the codes DETAIL and SURFACE (latest versions, Butler 1998, private communication).

It is interesting to note that we make a consistent synthesis for each star and element, for the particular physical conditions determined by the stellar parameters $T_{\text {eff }}, \log g$ and $\epsilon(\mathrm{He})$. Such a detailed analysis is not common in the literature. We have studied the methodologies followed in the works with which we will compare our results: Gies \& Lambert (1992), Cunha \& Lambert (1994), Schönberner et al. (1988) and Daflon et al. (1999) and only the latter two make a consistent synthesis of the metallic spectra.

Gies \& Lambert (1992) and Cunha \& Lambert (1994) make use of the NLTE metallic abundances of the Munich group, calculated with the LTE line-blanketed model atmospheres of Gold (1984), while the $\mathrm{H} / \mathrm{He}$ analysis was made with the LTE line-blanketed model atmospheres of Kurucz (1979), with a more complete blanketing than that of Gold (1984).
We feel that the additional effort is worthwhile in terms of consistency, specially for the relative abundances determined, which will hopefully remain stable whatever physical assumptions are changed in forthcoming works. Furthermore, Cunha \& Lambert (1994) find that using the same model atmospheres for the $\mathrm{H} / \mathrm{He}$ and the metallic synthesis consistently, apart from leading to different results, removes the dependence of the $\mathrm{C}$ and $\mathrm{N}$ abundances on the stellar temperature.

\subsection{The metallic line opacities}

When synthesizing the CNO spectra, we have made extensive use of the line opacities from the Kurucz opacity distribution functions (ODF), at solar abundance and $2 \mathrm{~km} \mathrm{~s}^{-1}$ microturbulence (Kurucz 1996).

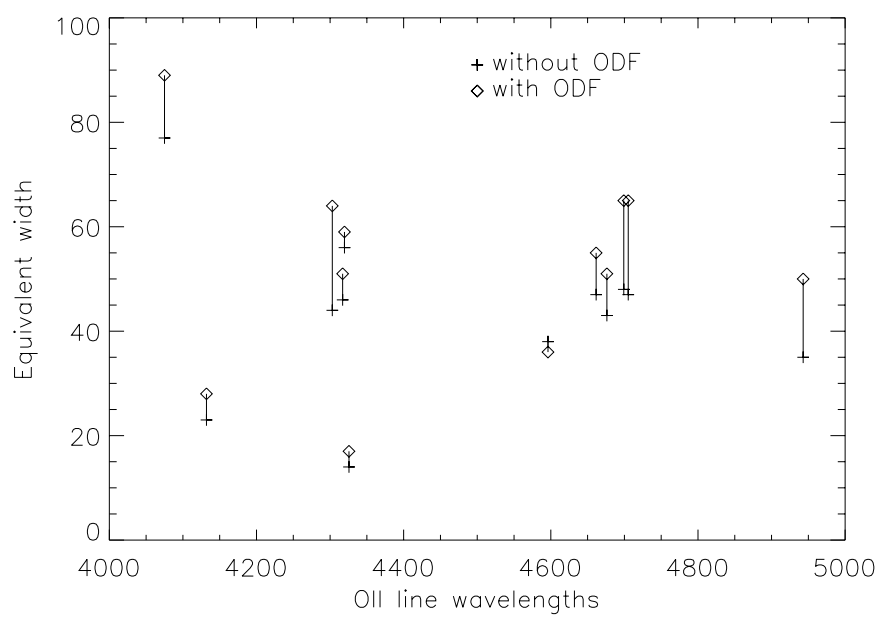

Fig. 2. Changes in the O II model equivalent widths (in $\mathrm{m} \AA$ ) due to the use of the ODFs of Kurucz (1996) in the spectral synthesis. Calculations are made at solar abundance and $0 \mathrm{~km} \mathrm{~s}^{-1}$ microturbulence, for the parameters of the star HD 34078. Line wavelengths are given in $\AA$.

This is to account for the stellar flux blocking due to the metallic lines, which produces important effects on the synthesized spectra, as can be seen in Fig. 2 for the particular case of O II in HD 34078. Therefore, the use of the ODFs will lead to more appropriate abundances.

For the $\mathrm{H} / \mathrm{He}$ spectral synthesis we have made use of a different set of metallic lines, from 228 to $912 \AA$ (see Herrero et al. 2000 for further details) instead of the longer range (9-100000 $\AA$ ) considered for CNO. However, in the former range we find the strongest contribution of lineblocking to the $\mathrm{H} / \mathrm{He}$ occupation numbers, so the new opacities considered would yield the same $\mathrm{H} / \mathrm{He}$ spectra.

\subsection{The model atoms}

The model atoms used are basically those described in Becker \& Butler (1988) for O II and in Eber (1987) for $\mathrm{C}$ III. We use a combined N II-N III model atom which 


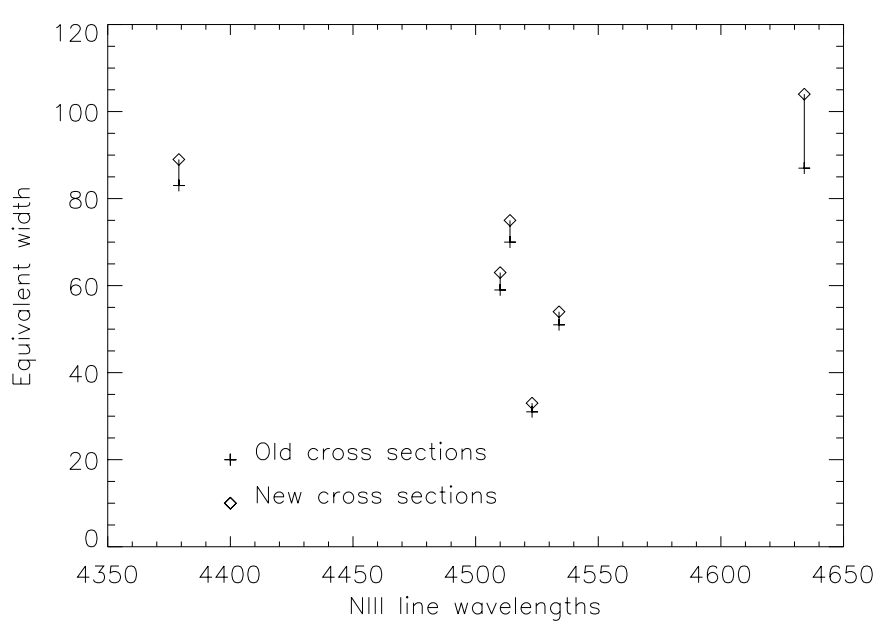

Fig. 3. Changes in the N III model equivalent widths (in $\mathrm{m} \AA$ ) due to the updated photoionization cross-sections (see text). Calculations are made at solar abundance and $0 \mathrm{~km} \mathrm{~s}^{-1} \mathrm{mi}-$ croturbulence, for the parameters of the star HD 214680. Line wavelengths are given in $\AA$.

was constructed using the N II model of Becker \& Butler (1989) and the N III model of Butler (1984).

We have included a few new transitions in the O II and C III model atoms (see Table 4) that were observed in our spectra and are not present in the quoted models. The N II and N III photoionization cross sections have been updated with the Opacity Project atomic data (Cunto et al. 1993). The effects of the new cross sections on the synthetic equivalent widths can be seen in Fig. 3 .

To calculate the Voigt profile of these transitions, we assume that the collisional broadening is much more important than the radiative natural broadening and omit the latter. We adopt the formula of Cowley (1971) for the collisional parameters. At the high temperatures of our models, this is a good assumption because Doppler broadening dominates the profiles.

During the abundance analysis we have excluded the C III 4673 line from the calculation of the $\mathrm{C}$ abundance of the three He normal stars, because it yields considerably higher abundances than the remainder of the lines. This indicates either that the line is not being well synthesized, or perhaps that the log gf value is underestimated. The rest of the new transitions give line abundances consistent with the "old" ones.

\subsection{Microturbulence}

A wealth of work in the literature (see Vrancken 1997 and McErlean et al. 1999 for example) show how the value of microturbulence can depend on which element is considered in a given atmosphere. The two previous works in particular show how, for B stars, the value obtained from the $\mathrm{O}$ II lines is systematically higher than that obtained from other ions, such as Si III.
Table 4. New transitions included in the atomic models of O II and C III. The level energies are taken from the NIST database (http://physics.nist.gov), in $\mathrm{cm}^{-1}$, and the $\log g f$ values from VALD. Only the CIII 4673 line has been excluded from the abundance determinations in the whole sample (see text).

\begin{tabular}{|c|c|c|c|}
\hline Trans. & $E_{1}$ & $E_{\mathrm{u}}$ & $\log g f$ \\
\hline C III 4056 & 324212.49 & 348859.99 & 0.265 \\
\hline C III 4067 & 322003.68 & 346579.21 & 0.719 \\
\hline C III 4068 & 322009.58 & 346579.21 & 0.837 \\
\hline C III 4068 & 322009.58 & 346579.21 & -0.340 \\
\hline C III 4070 & 322017.97 & 346579.49 & 0.952 \\
\hline C III 4070 & 322017.97 & 346579.21 & -0.340 \\
\hline C III 4070 & 322017.97 & 346579.21 & -2.157 \\
\hline C III 4186 & 322702.02 & 346579.31 & 0.918 \\
\hline C III 4325 & 310006.32 & 333118.21 & 0.137 \\
\hline C III 4379 & 321411.31 & 344238.68 & -1.130 \\
\hline C III 4379 & 321411.31 & 344236.29 & -1.255 \\
\hline C III 4380 & 321411.31 & 344232.98 & -2.431 \\
\hline C III 4382 & 321426.74 & 344236.29 & -0.778 \\
\hline C III 4383 & 321426.74 & 344232.98 & -1.255 \\
\hline C III 4388 & 321450.05 & 344232.98 & -0.507 \\
\hline C III 4515 & 317794.26 & 339934.72 & -0.757 \\
\hline C III 4515 & 317796.51 & 339934.72 & -0.280 \\
\hline C III 4516 & 317801.30 & 339934.72 & -0.058 \\
\hline C III 4647 & 238213.00 & 259724.30 & 0.007 \\
\hline C III 4665 & 308317.29 & 329743.57 & 0.044 \\
\hline C III 4673 & 308317.29 & 329706.47 & -0.434 \\
\hline O II 4253 & 252608.28 & 276109.54 & 0.936 \\
\hline O II 4253 & 252608.28 & 276109.46 & -0.804 \\
\hline O II 4253 & 252609.46 & 276109.46 & 0.846 \\
\hline O II 4602 & 234402.80 & 256125.78 & 0.510 \\
\hline
\end{tabular}

We take this into account and make independent determinations of the abundance together with the microturbulence value for each element.

In synthesizing the CNO spectra it is necessary to decide how to treat microturbulence, i.e., whether to include it in both the calculation of the populations and in the line formation (in both DETAIL and SURFACE), or only in the line formation (only in SURFACE).

Vrancken (1997) finds that for the high values of microturbulence of the giants of her sample, the inclusion in both DETAIL and SURFACE can lead to lower abundances by $0.1-0.2$ dex. As we determine differential abundances, we do not include microturbulence in DETAIL in the present work.

\section{Abundance uncertainties}

The determination of the abundance uncertainties is as important as the abundances themselves.

Our absolute abundances are determined from the mean value of the individual line abundances (each of them calculated for the mean microturbulence, see Sect. 5) of all the well behaved lines in the abundance-equivalent width diagrams (see Fig. 1). In order to consider all the possible sources of uncertainty in this measurement we proceed following Israelian et al. (1998). 
Table 5. Line uncertainties in the $\mathrm{C}$ III lines due to $\Delta T_{\mathrm{eff}}, \Delta \log g$ and $\Delta \epsilon(\mathrm{He})$, in logarithmic scale. $\Delta \log \epsilon_{\mathrm{i}}^{\text {mod }}$ is the total line uncertainty.

\begin{tabular}{cccccccccc}
\hline \hline Line & \multicolumn{2}{c}{$\Delta T_{\text {eff }}$} & \multicolumn{2}{c}{$\Delta \log g$} & \multicolumn{2}{c}{$\Delta \epsilon(\mathrm{He})$} & \multicolumn{2}{c}{$\Delta \log \epsilon_{\mathrm{i}}^{\text {mod }}$} & \multicolumn{2}{c}{$\Delta \log \epsilon_{\mathrm{i}}^{\text {mod }}$} \\
& $-1000 \mathrm{~K}$ & $+1000 \mathrm{~K}$ & $-0.10 \mathrm{dex}$ & $+0.10 \mathrm{dex}$ & -0.02 & +0.02 & - & + & \\
\hline C III 4156 & -0.07 & 0.09 & 0.16 & -0.07 & 0.01 & 0.00 & -0.09 & 0.18 & \pm 0.13 \\
C III 4162 & -0.04 & 0.10 & 0.16 & -0.06 & 0.01 & -0.01 & -0.08 & 0.19 & \pm 0.14 \\
C III 4186 & -0.04 & 0.09 & 0.12 & -0.05 & 0.00 & -0.01 & -0.08 & 0.15 & \pm 0.12 \\
C III 4665 & -0.11 & 0.21 & 0.36 & -0.11 & 0.02 & -0.02 & -0.16 & 0.42 & \pm 0.29 \\
& & \multicolumn{3}{c}{$\Delta \log \epsilon(\mathrm{C})^{\text {mod }}$} \\
\hline
\end{tabular}

Table 6. Same as Table 5 but now for N.

\begin{tabular}{cccccccccc}
\hline \hline Line & \multicolumn{2}{c}{$\Delta T_{\text {eff }}$} & \multicolumn{2}{c}{$\Delta \log g$} & \multicolumn{2}{c}{$\Delta \epsilon(\mathrm{He})$} & \multicolumn{2}{c}{$\Delta \log \epsilon_{\mathrm{i}}^{\text {mod }}$} & \multicolumn{2}{c}{$\Delta \log \epsilon_{\mathrm{i}}^{\text {mod }}$} \\
& $-1000 \mathrm{~K}$ & $+1000 \mathrm{~K}$ & $-0.10 \mathrm{dex}$ & $+0.10 \mathrm{dex}$ & -0.02 & +0.02 & - & + & \\
\hline N III 4510 & 0.18 & -0.08 & -0.06 & 0.08 & -0.00 & 0.02 & -0.20 & 0.10 & \pm 0.15 \\
N III 4514 & 0.19 & -0.10 & -0.08 & 0.07 & -0.01 & 0.01 & -0.20 & 0.13 & \pm 0.16 \\
N III 4523 & 0.11 & -0.08 & -0.05 & 0.04 & -0.01 & 0.01 & -0.12 & 0.10 & \pm 0.11 \\
& \multicolumn{7}{c}{$\Delta \log \epsilon(\mathrm{N})^{\bmod }$} \\
\hline
\end{tabular}

The standard deviation of the line abundances, when the number of lines is large enough and they are weak to strong, will give us the uncertainty from three different sources: first from the uncertainty in the position of the continuum, second from the mean value of microturbulence adopted for the individual abundances, and third from the limitations of the model atoms, or in fact of the entire spectral synthesis process.

The other sources of uncertainty come from the uncertainty in the stellar parameters, $\Delta T_{\text {eff }}, \Delta \log g$ and $\Delta \epsilon(\mathrm{He})$ ( $\pm 1000 \mathrm{~K}, \pm 0.1$ dex and $\pm 25 \%$ respectively, see Villamariz 2001).

Villamariz (2001) has shown how H/He model spectra of lower gravities, those suitable for supergiants, are the most sensitive to changes in the model calculations. We expect this also to apply to the metallic spectra, so we estimate the line abundance uncertainties for our supergiant HD 209975 and take them as maximum values of the uncertainty for the whole O9 sample.

The stellar parameters of HD 209975 are: $T_{\text {eff }}=$ $34000 \mathrm{~K}, \log g=3.20$ and $\epsilon(\mathrm{He})=0.08$. For the calculation of the line uncertainties we proceed as follows. We construct a grid of six model atmospheres (and $\mathrm{H} / \mathrm{He}$ populations), putting each parameter in turn to one of its two extreme values and fixing the other two parameters to the central values. With each model of the grid we perform all the abundance calculations as for the central parameters, to obtain new line abundances (see Tables 5 to 7 ).

In this way we calculate the line uncertainties due to each parameter independently, and combine them quadratically to find the final line uncertainties due to the errors in the stellar parameters $\left(\Delta \epsilon_{\mathrm{i}}^{\bmod }\right)$. In particular, we have taken the contributions towards higher and lower abundances separately:

$\Delta \epsilon_{\mathrm{i}}^{\bmod \pm}=\sqrt{\Delta^{2} \epsilon_{\mathrm{i}}^{T_{\text {eff }} \pm}+\Delta^{2} \epsilon_{\mathrm{i}}^{\log \mathrm{g} \pm}+\Delta^{2} \epsilon_{\mathrm{i}}^{\epsilon(\mathrm{He}) \pm}}$, and $\Delta \epsilon_{\mathrm{i}}^{\mathrm{mod}}$ is the mean of those two values (see Tables 5 to 7).

Again following Israelian et al. (1998), these individual uncertainties are used to compute the mean uncertainty for each element $\left(\Delta \epsilon(\mathrm{X})^{\text {mod }}\right)$, which is then quadratically combined with the standard deviation to give the final uncertainty in the absolute abundance:

$\Delta \epsilon(\mathrm{X})=\sqrt{\sigma^{2}+\Delta^{2} \epsilon^{\mathrm{mod}}}$.

Remember that while the standard deviation in the line abundances is individually calculated for each star and element, the uncertainty due to the model parameters is the same for the whole sample.

\section{Absolute abundances of the He normal stars}

We begin the abundance analysis by determining preliminary absolute abundances and microturbulence values for each element as explained in Sect. 3. The line abundanceequivalent width diagrams give us the set of lines suitable for the abundance determination, according to the dispersion found in each case.

With the mean value of microturbulence given by the values found for the three elements, we compute the final line abundances and absolute abundances, given in Table 11. The final absolute abundances are the mean of the individual values, and its uncertainty is the combination of the standard deviation and the error coming from the stellar parameters as explained in Sect. 4.

Below we give details on the preliminary abundance determination for each star, and then we give the final absolute abundances for the three stars in Table 11. 
Table 7. Same as Table 5 but now for O.

\begin{tabular}{|c|c|c|c|c|c|c|c|c|c|}
\hline \multirow[t]{2}{*}{ Line } & \multicolumn{2}{|c|}{$\Delta T_{\text {eff }}$} & \multicolumn{2}{|c|}{$\Delta \log g$} & \multicolumn{2}{|c|}{$\Delta \epsilon(\mathrm{He})$} & \multicolumn{2}{|c|}{$\Delta \log \epsilon_{\mathrm{i}}^{\bmod }$} & \multirow[t]{2}{*}{$\Delta \log \epsilon_{\mathrm{i}}^{\mathrm{mod}}$} \\
\hline & $-1000 \mathrm{~K}$ & $+1000 \mathrm{~K}$ & -0.10 dex & $+0.10 \mathrm{dex}$ & -0.02 & +0.02 & - & + & \\
\hline O II 4075 & -0.13 & 0.21 & 0.24 & -0.09 & -0.00 & -0.00 & -0.16 & 0.33 & \pm 0.24 \\
\hline О Іі 4282 & -0.11 & 0.19 & 0.24 & -0.10 & 0.01 & -0.00 & -0.15 & 0.30 & \pm 0.22 \\
\hline O II 4294 & -0.12 & 0.21 & 0.21 & -0.12 & -0.00 & -0.00 & -0.17 & 0.29 & \pm 0.23 \\
\hline О іг 4303 & -0.09 & 0.21 & 0.24 & -0.06 & 0.04 & -0.00 & -0.11 & 0.32 & \pm 0.21 \\
\hline O II 4327 & -0.16 & 0.27 & 0.35 & -0.13 & 0.03 & -0.01 & -0.21 & 0.45 & \pm 0.33 \\
\hline О іг 4349 & -0.11 & 0.19 & 0.16 & -0.06 & 0.00 & 0.01 & -0.13 & 0.24 & \pm 0.19 \\
\hline О іг 4366 & -0.15 & 0.27 & 0.33 & -0.10 & 0.01 & 0.00 & -0.18 & 0.42 & \pm 0.30 \\
\hline О II 4414 & -0.22 & 0.56 & 1.02 & -0.19 & 0.04 & -0.01 & -0.29 & 1.16 & \pm 0.72 \\
\hline О іг 4602 & -0.10 & 0.16 & 0.20 & -0.06 & -0.00 & -0.00 & -0.11 & 0.26 & \pm 0.19 \\
\hline О іг 4609 & -0.11 & 0.16 & 0.19 & -0.09 & -0.00 & -0.00 & -0.15 & 0.25 & \pm 0.20 \\
\hline O II 4638 & -0.13 & 0.16 & 0.14 & -0.08 & -0.02 & -0.00 & -0.15 & 0.22 & \pm 0.18 \\
\hline О іг 4661 & -0.13 & 0.19 & 0.16 & -0.07 & -0.00 & -0.00 & -0.15 & 0.25 & \pm 0.20 \\
\hline О іг 4705 & -0.13 & 0.19 & 0.26 & -0.10 & -0.00 & -0.00 & -0.16 & 0.32 & \pm 0.24 \\
\hline \multicolumn{9}{|c|}{$\Delta \log \epsilon(\mathrm{O})^{\bmod }$} & \pm 0.30 \\
\hline
\end{tabular}

Table 8. Preliminary absolute abundances for HD 214680, O9 V. For each element $n$ is the number of lines used, $\xi$ is the microturbulence and $\log \epsilon(\mathrm{X})$ is the logarithmic abundance by number relative to the total $\mathrm{H}+\mathrm{He}$. The uncertainties given here are $\sigma / \sqrt{n}$.

\begin{tabular}{|c|c|c|}
\hline $\bar{C}$ & $\overline{\mathrm{N}}$ & $\bar{O}$ \\
\hline $\begin{array}{l}\xi \quad n \\
\log \epsilon(\mathrm{C})\end{array}$ & $\begin{array}{l}\xi \quad n \\
\log \epsilon(\mathrm{N})\end{array}$ & $\begin{array}{l}\xi \\
\log \epsilon(\mathrm{O})\end{array}$ \\
\hline $\begin{array}{ll}22.5 & 7\end{array}$ & $\begin{array}{ll}10 & 4\end{array}$ & $22.5 \quad 23$ \\
\hline$-3.97 \pm 0.06$ & $-4.11 \pm 0.05$ & $-3.38 \pm 0.04$ \\
\hline
\end{tabular}

\subsection{HD 214680 (10 Lac), O9 V}

This star belongs to Lac OB1, an association at 368 pc from the Sun according to the Hipparcos data (de Zeeuw et al. 1999). Kane et al. (1980) have measured LTE abundances for two B2 stars of the association, and Gies \& Lambert (1992) and Schönberner et al. (1988) for HD 214680.

We only have a few lines for each element in common with Gies \& Lambert (1992), and the comparison with our equivalent widths shows that theirs are 5 to $30 \%$ lower than ours. However, this difference is what they find when comparing their values to the literature, and they argue that the reason must be their underestimation of the far wings of the line when computing the equivalent width.

Schönberner et al. (1988) have a total of nine lines of $\mathrm{CNO}$ in common with us, and even though their spectra are photographic and with a lower $S / N$, there is good agreement between our respective values.

The preliminary absolute abundances of $\mathrm{CNO}$ for this star are given in Table 8. The microturbulence of $22.5 \mathrm{~km} \mathrm{~s}^{-1}$ indicated by the $\mathrm{O}$ II lines is not a good value. These lines are too weak to be sensitive to microturbulence (the maximum equivalent width is only $93 \mathrm{~m} \AA$, see
Table 3), and the scatter in the line abundances is not minimum for this value of microturbulence, so we do not take it as being representative.

Note that O II in spite of being the most numerous ion in the spectrum has an uncertainty in the preliminary absolute abundance of the order of those for $\mathrm{C}$ and $\mathrm{N}$, showing how the $\mathrm{O}$ II line abundances are more scattered than for the other two elements.

The high value of microturbulence also found for $\mathrm{C}$ is an uncomfortable result. Kudritzki (1992) and Lamers \& Achmad (1994) justify the high values found for supergiants as a result of the presence of stellar winds, but in the case of this dwarf star, without evidence in the spectrum of a strong wind, we cannot attribute this high value of microturbulence to this.

With the microturbulence indicated by $\mathrm{N}$ and $\mathrm{C}$ we find a mean microturbulence of $16 \pm 9 \mathrm{~km} \mathrm{~s}^{-1}$, with which we compute the final line and absolute abundances.

\section{2. $H D 34078,09.5 \mathrm{~V}$}

This star belongs to Ori OB1, which is located at about 500 pc from the Sun (de Zeeuw et al. 1999). Gies \& Bolton (1986) classify this star as a runaway, with its origin in OriOB1 and an actual peculiar radial velocity of $50 \mathrm{~km} \mathrm{~s}^{-1}$.

Ori OB1 is a very well known association, with a wealth of abundance studies such as those of Kilian (1992), Cunha \& Lambert $(1992,1994)$ and Cunha et al. (1998). They all determine abundances of early B dwarf stars, with which we compare our results.

Gies \& Lambert (1992) have determined abundances for this star. As for HD 214680 our equivalent widths are higher than theirs, due to the reasons stated.

The preliminary absolute abundances of CNO for this star are given in Table 9. The microturbulence of 
Table 9. As in table 8 for HD 34078, O9.5 V.

\begin{tabular}{|c|c|c|}
\hline$\overline{\mathrm{C}}$ & $\overline{\mathrm{N}}$ & $\overline{\mathrm{O}}$ \\
\hline $\begin{array}{l}\xi \quad n \\
\log \epsilon(\mathrm{C})\end{array}$ & $\begin{array}{l}\xi \\
\log \epsilon(\mathrm{N})\end{array}$ & $\begin{array}{l}\xi \\
\log \epsilon(\mathrm{O})\end{array}$ \\
\hline $12.5 \quad 6$ & $15 \quad 4$ & $17.5 \quad 23$ \\
\hline$-3.82 \pm 0.05$ & $-4.18 \pm 0.09$ & $-3.37 \pm 0.05$ \\
\hline
\end{tabular}

$17.5 \mathrm{~km} \mathrm{~s}^{-1}$ indicated by the $\mathrm{O}$ II lines is again disregarded for the same reasons argued for HD 214680. And again, the line abundances for $\mathrm{O}$ show more scatter than for $\mathrm{C}$ and $\mathrm{N}$.

With the microturbulence indicated by $\mathrm{N}$ and $\mathrm{C}$ we obtain a mean microturbulence of $14 \pm 2 \mathrm{~km} \mathrm{~s}^{-1}$, with which we compute the final absolute abundances.

\section{3. $H D 209975,09.5 \mathrm{lb}$}

This star belongs to Cep OB2, an association at 615 pc from the Sun (de Zeeuw et al. 1999). Daflon et al. (1999) have measured abundances for dwarfs from O9 to B2 in this association.

HD 209975 has a higher rotational velocity than the two dwarfs studied, and so its lines are more blended and diluted, leading to higher uncertainties in the equivalent widths and to a lower number of isolated lines suitable for the abundance analysis (see Tables 2 and 3).

Table 10. As in table 8 for HD 209975, O9.5 Ib.

\begin{tabular}{|c|c|c|}
\hline $\mathrm{C}$ & $\mathrm{N}$ & $\mathrm{O}$ \\
\hline $\begin{array}{l}\xi \quad n \\
\log \epsilon(\mathrm{C})\end{array}$ & $\begin{array}{l}\xi \quad \begin{array}{r}n \\
\log \epsilon(\mathrm{N})\end{array}\end{array}$ & $\begin{array}{l}\xi \\
\log \epsilon(\mathrm{O})\end{array}$ \\
\hline $12.5 \quad 4$ & $\begin{array}{ll}25 & 3\end{array}$ & $>25 \quad 13$ \\
\hline$-4.03 \pm 0.06$ & $-3.97 \pm 0.01$ & $<-3.63 \pm 0.12$ \\
\hline
\end{tabular}

While the set of lines finally excluded for the abundance determination in the two dwarfs were coincident for C III and N III, and only differ in a single line for O II, in the case of the supergiant HD 209975 we find that the set of lines that must be excluded according to their dispersion in the abundance-equivalent width diagrams, are not completely coincident. To assure consistency in our analysis, we decided to exclude both sets of lines for every element, so that only those lines with suitable scatter and also considered in the two dwarfs contribute to the abundance determination.

This leads to the preliminary abundances in Table 10. The scatter in the O II line abundances is especially severe in this star: compare the $\sigma / \sqrt{n}$ values for the dwarfs in Tables 8 and 9 of 0.04 and 0.05 , to the 0.12 value for HD 209975. Again the microturbulence value indicated by $\mathrm{O}$ is not considered to be representative.

With a mean microturbulence of $\xi=20 \pm 10 \mathrm{~km} \mathrm{~s}^{-1}$ we compute the final absolute abundances for HD 209975 given in Table $11\left(\log \epsilon_{\mathrm{i} 1}\right)$. As seen in Villamariz \& Herrero (2000), the use of a value of $\xi=15 \mathrm{~km} \mathrm{~s}^{-1}$ for the $\mathrm{H} / \mathrm{He}$ spectral synthesis of models suitable for supergiants can lead to changes in the stellar parameters of the order of our standard error box of $1000 \mathrm{~K}$ in $T_{\text {eff }}, 0.1$ dex in $\log g$ and $25 \%$ in $\epsilon(\mathrm{He})$. The changes in the stellar parameters influence the line abundances determined as explained in Sect. 4 , so we apply the correspondent corrections to these abundances due to the value of microturbulence found for this star.

The changes in the parameters are adopted from those found for the O9 supergiant HD 210809 in Villamariz \& Herrero (2000): $\Delta T_{\text {eff }}=+1000 \mathrm{~K}, \Delta \log g=+0.05$ dex and $\Delta \epsilon(\mathrm{He})=-20 \%$. With them we compute the line abundances of the second column in Table 11 for HD 209975, which are the final values. As seen in the table these results are more consistent with the two dwarfs HD 214680 and HD 34078.

\section{Relative abundances of the He normal stars}

As explained in Sect. 2, computing differential line abundances will give us the best estimates of the differences in the chemical compositions of the stars studied. For stars of similar parameters, and therefore similar physical conditions, the systematic errors in the line abundances will be the same for all stars. The errors will thus be reduced by the use of the differential method.

Among the three He normal stars, the two dwarfs HD 214680 and HD 34078, with projected rotational velocities of 50 and $40 \mathrm{~km} \mathrm{~s}^{-1}$ respectively, are assumed to provide the reference values for the abundances of CNO in unmixed stars. A comparison of their abundances will give information about the consistency of our method, which should yield similar values for both, and therefore also about the scatter that can be expected between stars of similar chemical compositions. Remember that both stars are in the solar neighbourhood (de Zeeuw et al. 1999), so they are not expected to have had different initial chemical compositions.

The supergiant HD 209975, also from the solar neighbourhood and with $v \sin i=100 \mathrm{~km} \mathrm{~s}^{-1}$, is assumed to provide the CNO reference abundances for stars of lower gravities. The differential analysis to the dwarf stars will then tell us whether its slightly different physical conditions, basically the more extensive atmosphere with lower surface gravity, introduces any changes in the reference CNO abundances.

The material of this star should not be mixed. Its He abundance is normal and its rotational velocity is lower than the $200 \mathrm{~km} \mathrm{~s}^{-1}$ considered as the approximate initial minimum value of the surface equatorial rotation for efficient mixing (Meynet \& Maeder 2000). But we must keep in mind that the $100 \mathrm{kms}^{-1}$ is a projected value, and also that the initial rotational velocities of massive stars decrease due to the loss of angular momentum in the stellar wind (Meynet \& Maeder 2000; Heger \& Langer 2000), so the hypothesis of unmixed material must be checked. In adittion, a normal atmospheric He abundance is compatible with increments in the $\mathrm{N} / \mathrm{C}$ and $\mathrm{N} / \mathrm{O}$ ratios in 
Table 11. Final line abundances and absolute abundances for the three He normal stars. Equivalent widths are also given. For HD 209975 we give two sets of line abundances: $\log \epsilon_{\mathrm{i} 2}$ are the final values, corrected for the effect of microturbulence in the stellar parameters (see text).

\begin{tabular}{|c|c|c|c|c|c|c|c|}
\hline \multirow[t]{2}{*}{ Line } & \multicolumn{2}{|c|}{ HD 214680} & \multicolumn{2}{|c|}{ HD 34078} & \multicolumn{3}{|c|}{ HD 209975} \\
\hline & $E W$ & $\log \epsilon_{\mathrm{i}}$ & $E W$ & $\log \epsilon_{\mathrm{i}}$ & $E W$ & $\log \epsilon_{\mathrm{i} 1}$ & $\log \epsilon_{\mathrm{i} 2}$ \\
\hline C III 4056 & 34 & -4.03 & - & - & - & - & - \\
\hline C III 4156 & 64 & -3.79 & 64 & -3.78 & 26 & -4.12 & -4.07 \\
\hline C III 4162 & 64 & -3.94 & 82 & -3.78 & 34 & -4.14 & -4.07 \\
\hline C III 4186 & 99 & -4.11 & 122 & -3.92 & 98 & -4.17 & -4.11 \\
\hline C III 4325 & 33 & -4.15 & 51 & -3.95 & - & - & - \\
\hline C III 4647 & 268 & -3.66 & 267 & -3.63 & - & - & - \\
\hline C III 4665 & 55 & -3.77 & 51 & -3.86 & 35 & -3.92 & -3.77 \\
\hline $\log \epsilon(\mathrm{C})$ & \multicolumn{2}{|c|}{$-3.89 \pm 0.22$} & \multicolumn{2}{|c|}{$-3.81 \pm 0.16$} & \multicolumn{2}{|c|}{$-4.08 \pm 0.23$} & $-3.98 \pm 0.24$ \\
\hline N III 4379 & 105 & -4.39 & 84 & -4.39 & - & - & - \\
\hline N III 4510 & 84 & -4.13 & 62 & -4.18 & 136 & -3.94 & -4.00 \\
\hline N III 4514 & 106 & -4.11 & 109 & -3.91 & 16 & -3.89 & -3.98 \\
\hline N III 4523 & 37 & -4.16 & 34 & -4.05 & 59 & -3.96 & -4.04 \\
\hline $\log \epsilon(\mathrm{N})$ & \multicolumn{2}{|c|}{$-4.19 \pm 0.28$} & \multicolumn{2}{|c|}{$-4.10 \pm 0.28$} & \multicolumn{2}{|c|}{$-3.93 \pm 0.15$} & $-4.01 \pm 0.17$ \\
\hline О Іі 4072 & 93 & -3.35 & 92 & -3.56 & - & - & - \\
\hline O II 4075 & 87 & -3.61 & 104 & -3.67 & 78 & -3.75 & -3.61 \\
\hline O Іі 4087 & 40 & -3.49 & 32 & -3.76 & - & - & - \\
\hline O II 4132 & 31 & -3.07 & - & - & - & - & - \\
\hline O II 4189 & 36 & -3.48 & 58 & -3.44 & - & - & - \\
\hline O II 4253 & 73 & -3.08 & 85 & -3.18 & - & - & - \\
\hline О пі 4282 & 31 & -3.70 & - & - & 39 & -3.49 & -3.36 \\
\hline O Іі 4288 & 20 & -3.16 & 28 & -3.15 & - & - & - \\
\hline O II 4294 & 35 & -3.34 & 39 & -3.42 & 6 & -4.11 & -3.99 \\
\hline O II 4303 & 34 & -3.67 & 61 & -3.49 & 12 & -4.12 & -3.94 \\
\hline O Іі 4317 & - & - & 89 & -3.08 & - & - & - \\
\hline O пі 4319 & - & - & 74 & -3.29 & - & - & - \\
\hline O II 4327 & 17 & -3.22 & 38 & -3.03 & 13 & -3.15 & -2.97 \\
\hline О Іі 4349 & 54 & -3.70 & 75 & -3.70 & 32 & -4.15 & -4.02 \\
\hline О пі 4366 & 65 & -3.14 & 79 & -3.23 & 72 & -3.15 & -2.97 \\
\hline О іл 4369 & - & - & 28 & -3.15 & - & - & - \\
\hline O II 4414 & 47 & -3.60 & 67 & -3.69 & 25 & -3.66 & -3.32 \\
\hline O Іі 4596 & 27 & -3.25 & 40 & -3.42 & - & - & - \\
\hline O II 4602 & 34 & -3.42 & 32 & -3.63 & 8 & -4.11 & -3.99 \\
\hline О пі 4609 & 69 & -3.24 & - & - & 41 & -3.50 & -3.40 \\
\hline O Іі 4638 & 66 & -3.04 & 90 & -3.04 & 19 & -3.99 & -3.90 \\
\hline O іп 4661 & 53 & -3.33 & 63 & -3.44 & 24 & -4.01 & -3.88 \\
\hline O II 4676 & 46 & -3.33 & 62 & -3.36 & - & - & - \\
\hline О іг 4699 & 40 & -3.65 & 63 & -3.61 & - & - & - \\
\hline O Іі 4705 & 46 & -3.60 & 51 & -3.75 & 16 & -4.00 & -3.88 \\
\hline O II 4943 & 21 & -3.63 & 40 & -3.56 & - & - & - \\
\hline $\log \epsilon(\mathrm{O})$ & -3.35 & \pm 0.27 & -3.36 & \pm 0.29 & -3.6 & \pm 0.52 & $-3.46 \pm 0.48$ \\
\hline
\end{tabular}

the first stages of the main sequence evolution (Heger \& Langer 2000).

In Table 12 we give the line by line differential analysis of the three He normal stars. The final relative abundances are the simple mean of the individual values, and the standard deviation its corresponding uncertainty.
This is what we find:

- The two dwarf stars have CNO abundances indistinguishable within the uncertainties, with a scatter in the CN values of 0.1 dex (with HD 34078 more abundant than HD 214680) and the same O abundance. 
Table 12. Line by line differential abundances of the three $\mathrm{He}$ normal stars.

\begin{tabular}{lcc}
\hline \hline Line & HD 34078 & HD 209975 \\
& vs. & vs. \\
& HD 214680 & HD 214680 \\
\hline C III 4156 & +0.02 & -0.28 \\
C III 4162 & +0.16 & -0.13 \\
C III 4186 & +0.20 & +0.00 \\
C III 4325 & +0.20 & - \\
C III 4647 & +0.03 & - \\
C III 4665 & -0.08 & +0.00 \\
\hline$\Delta$ log $\epsilon(\mathrm{C})$ & $+0.10 \pm 0.12$ & $-0.09 \pm 0.12$ \\
\hline N III 4379 & +0.00 & - \\
N III 4510 & -0.04 & +0.13 \\
N III 4514 & +0.20 & +0.13 \\
N III 4523 & +0.11 & +0.12 \\
\hline$\Delta$ log $\epsilon$ (N) & $+0.08 \pm 0.12$ & $+0.13 \pm 0.01$ \\
\hline O II 4072 & -0.21 & - \\
O II 4075 & -0.05 & -0.00 \\
O II 4087 & -0.26 & - \\
O II 4189 & +0.04 & - \\
O II 4253 & -0.09 & - \\
O II 4282 & - & -0.34 \\
O II 4288 & +0.01 & - \\
O II 4294 & -0.07 & -0.65 \\
O II 4303 & +0.18 & -0.27 \\
O II 4349 & +0.19 & -0.32 \\
O II 4366 & +0.00 & +0.17 \\
O II 4327 & -0.08 & +0.25 \\
O II 4414 & -0.09 & +0.28 \\
O II 4596 & -0.17 & - \\
O II 4602 & -0.20 & -0.57 \\
O II 4609 & - & -0.16 \\
O II 4638 & +0.00 & -0.86 \\
O II 4661 & -0.11 & -0.55 \\
O II 4676 & -0.03 & - \\
O II 4699 & +0.04 & - \\
O II 4705 & -0.15 & -0.28 \\
O II 4943 & +0.07 & $-0.05 \pm 0.36$ \\
\hline$\Delta$ log $\epsilon(\mathrm{O})$ & $-0.03 \pm 0.13$ & \\
\hline & &
\end{tabular}

- The supergiant HD 209975 also has CNO abundances that are indistinguishable from those of the dwarfs. The scatter in CN is also 0.1 dex and $\mathrm{O}$ abundance is again the same. It must be noted however, that the dispersion of the $\mathrm{CN}$ abundances goes in the direction of the $\mathrm{CNO}$ contamination: lower $\mathrm{C}$ abundance together with higher $\mathrm{N}$ abundance (see also Figs. 11 and 12).

\section{The fast rotator HD 191423}

This star belongs to the Cyg OB8 association, with a distance modulus of $11.8 \mathrm{mag}$ (Humphreys 1978) that situates it at $2.3 \mathrm{kpc}$ from the Sun. HD 191423 is a very fast rotator, $v \sin i=450 \mathrm{~km} \mathrm{~s}^{-1}$, with the weak metallic lines completely blended, so the method used for the other three stars cannot be used in this case (see Fig. 4). We perform a spectral synthesis and as we analyse the $\mathrm{H} /$ He spectra, fitting our model lines to the observed ones to find the stellar parameters $T_{\text {eff }}, \log g$ and $\epsilon(\mathrm{He})$, we find the best fits to the observed blends that give us the stellar CNO abundances. This methodology is widely used in stellar abundance determinations when the lines available are not isolated (see for example Pauldrach et al. 1994 and Taresch et al. 1997 for early O stars, and García-López et al. 1995 and Israelian et al. 1998 for late type stars).

We have identified all the metallic spectral features present in the spectrum of the star. Those that correspond to lines used for the three He normal stars are now selected for the abundance analysis. See Fig. 4 for the four metallic spectral features available:
(1): C III-O II 4067-82
(2): C III-N III-O II 4379
(3): C III-N III 4505-21
(4): C III-N III-O II 4620-60.

We have made a careful search of all the possible contributions to these blends, as explained in Sect. 3 when identifying lines in the stellar spectra. We find that the dominant contributions to each blend are those quoted, considering the lines that we have found in the other three stars in the same ranges, and also the synthetic spectra (see Sect. 7.2). We cannot make a line by line differential abundance analysis as for the other three stars, but for the fast rotator we make use of the lines that were suitable for the abundance determinations in the reference stars.

Blend (4) is in emission in Of stars together with the He II 4686 line (Walborn 1971). In the case of stars with weaker winds, like HD 191423, it can show some filling by the wind emission even while being in absorption, so we do not expect to obtain good fits for this blend.

In order to synthesize the complete $\mathrm{CNO}+\mathrm{H} / \mathrm{He}$ spectrum we proceed as follows. First we calculate individually the populations of each element, making use of DETAIL and the model atoms quoted in Sect. 3.2. Then we use a combined $\mathrm{CNO}+\mathrm{H} / \mathrm{He}$ model atom for the line formation calculations with SURFACE to reproduce the blends observed.

The last step in this process, before the comparison with the stellar spectrum, is the convolution of the synthetic profiles with a standard rotational broadening function (and of course with the instrumental profile, negligible in this case). This procedure is not very suitable for objects with such high rotational velocities, where surface deformation and gravity darkening become of considerable importance. Further work must be done to improve our treatment of rotation.

\subsection{Microturbulence and new stellar parameters}

As the entire $\mathrm{CNO}+\mathrm{H} / \mathrm{He}$ spectrum is calculated simultaneously, we have to make use of one particular value of microturbulence for all the elements. We consider the mean value found for the supergiant HD 209975, $\xi=20 \mathrm{~km} \mathrm{~s}^{-1}$, because of its similarity with our fast rotator.

As seen for HD 209975, this value of the microturbulence for a giant star like HD 191423 can lead to changes 


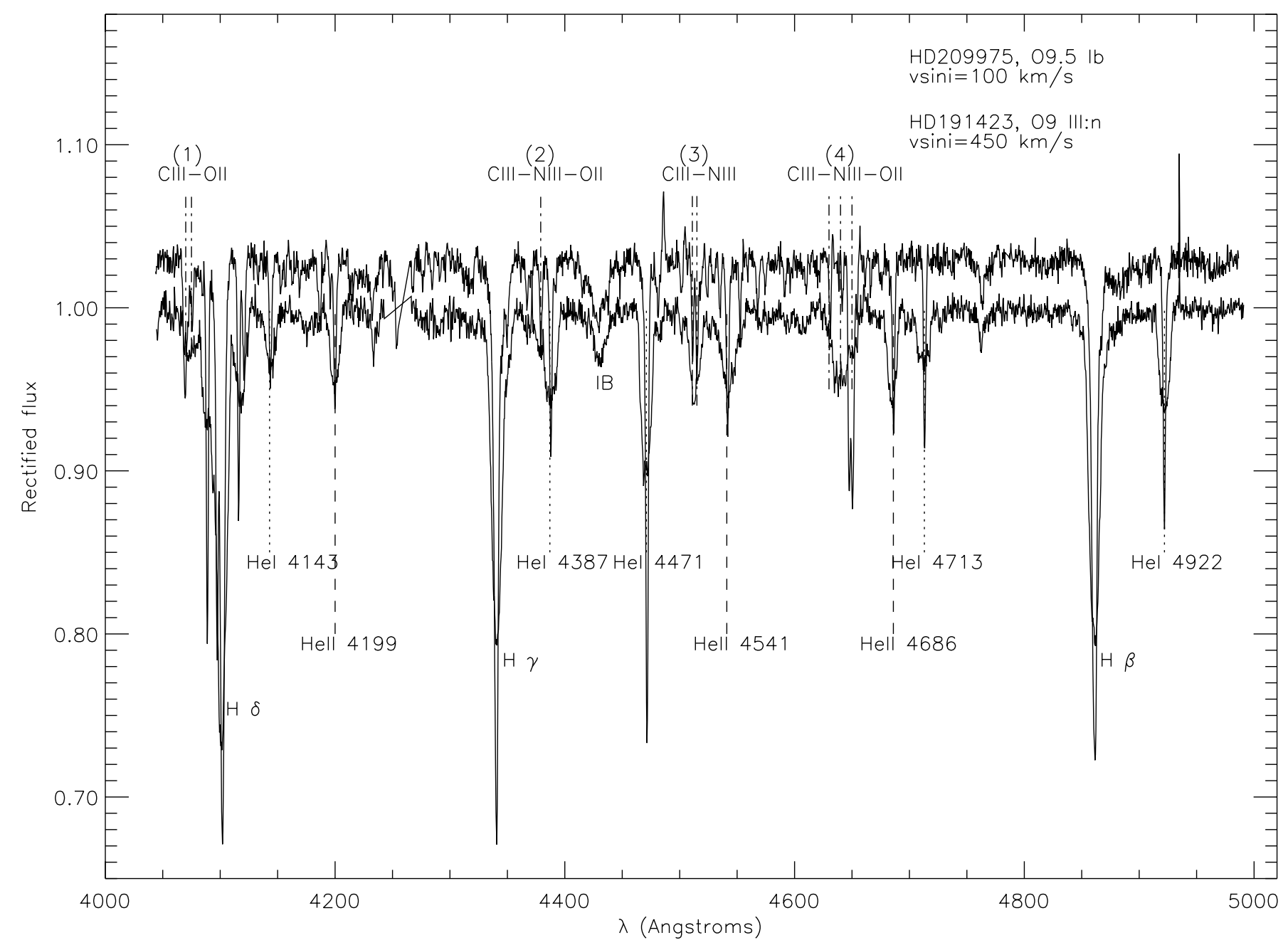

Fig. 4. Spectra of HD 191423 and HD 209975 (shifted for clarity). Compare how the lines of the fast rotator are blended while in HD 209975 can be still detached. IB stands for interstellar band.

in the stellar parameters, which have been determined for $\xi=0 \mathrm{~km} \mathrm{~s}^{-1}$. Therefore we proceed to determine new stellar parameters $T_{\text {eff }}, \log g$ and $\epsilon(\mathrm{He})$ at $\xi=20 \mathrm{~km} \mathrm{~s}^{-1}$.

We start with a model spectrum at $\xi=20 \mathrm{~km} \mathrm{~s}^{-1}$ and the initial parameters of the star: $T_{\text {eff }}=34000 \mathrm{~K}$, $\log g=3.40$ and $\epsilon(\mathrm{He})=0.20$ (see Fig. 5, dotted line) and we compute new model spectra in order to find the best fit for the observed $\mathrm{H} / \mathrm{He}$ lines (for details on the lines selected for the H/He analysis, see Herrero et al. 1992 and Villamariz 2001).

In Fig. 5 we see the final stage of the fitting, yielding the new parameters: $T_{\text {eff }}=35000 \mathrm{~K}, \log g=3.40, \epsilon(\mathrm{He})=$ 0.12 and $\xi=20 \mathrm{~km} \mathrm{~s}^{-1}$ (dash-dotted). It is interesting to note in the figure how the lines He I 4387 and He II 4541 cannot be well reproduced with the $\mathrm{H} / \mathrm{He}$ model atom, since they are blended with metallic lines (see Fig. 6). In the following spectral synthesis we see how the fits improve with the use of the combined $\mathrm{CNO}+\mathrm{H} / \mathrm{He}$ model atom (see Figs. 7 and 8). The blend of He II 4541 with Si III 4542 is not reproduced by this combined model atom.

With the new parameters He I 4471 shows the dilution effect present in the spectra of late $\mathrm{O}$ and early $\mathrm{B}$ giants and supergiants (see Voels et al. 1989; McErlean et al. 1998; Herrero et al. 1992), and He II 4686 shows some filling with wind emission.

On the basis of these new parameters we compute all the $\mathrm{CNO}+\mathrm{H} / \mathrm{He}$ synthetic spectra.

\subsection{CNO abundances in HD 191423, O9 III:n}

To start the abundance analysis of the fast rotator HD 191423 we begin with the abundances found for the reference supergiant HD 209975:

$\log \epsilon(\mathrm{C})=-3.98 \pm 0.24 \sim \log \frac{\epsilon(\mathrm{C})_{\odot}}{5}=-4.19$
$\log \epsilon(\mathrm{N})=-4.01 \pm 0.17 \sim \log \epsilon(\mathrm{N})_{\odot}=-4.07$
$\log \epsilon(\mathrm{O})=-3.46 \pm 0.48 \sim \log \frac{\epsilon(\mathrm{O})_{\odot}}{5}=-3.87^{1}$

With the CNO abundances of our standard grid of 10,5 and 2 times higher and lower than solar that are closest to

${ }^{1}$ In fact this value is closer to $\log \frac{\epsilon(\mathrm{O})_{\odot}}{2}=-3.47$, but a transcription error made us start with the quoted value. Nevertheless our results are not dependent on this. Solar abundances are taken from Grevesse et al. (1996). 

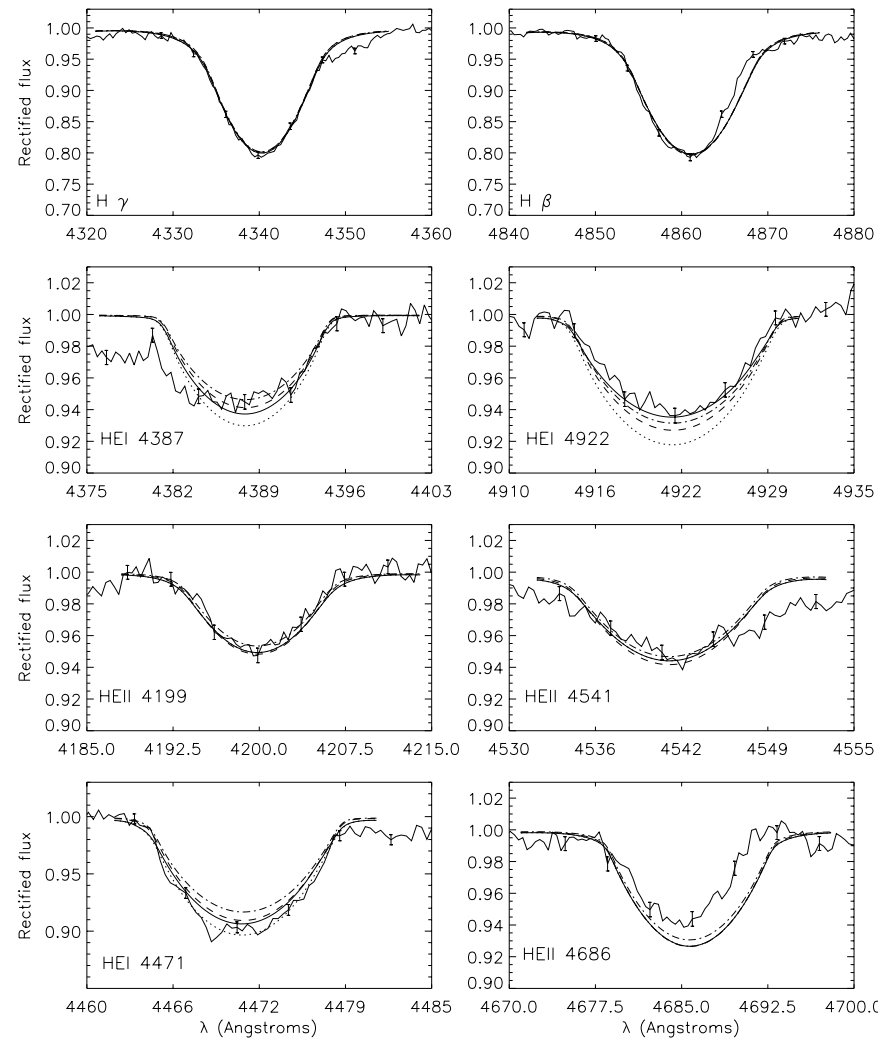

Fig. 5. Fitting the $\mathrm{H} / \mathrm{He}$ spectrum of HD 191423 to determine $T_{\text {eff }}, \log g$ and $\epsilon(\mathrm{He})$ at $\xi=20 \mathrm{~km} \mathrm{~s}^{-1}$. The initial parameters are $T_{\text {eff }}=34000 \mathrm{~K}, \log g=3.40, \epsilon(\mathrm{He})=0.20$, $\xi=0 \mathrm{~km} \mathrm{~s}^{-1}$ (solid line), then we plot the same model spectrum at $\xi=20 \mathrm{kms}^{-1}$ (dotted), and $T_{\text {eff }}=35000 \mathrm{~K}$, $\log g=3.40, \epsilon(\mathrm{He})=0.15, \xi=20 \mathrm{~km} \mathrm{~s}^{-1}$ (dashed). The final parameters are: $T_{\text {eff }}=35000 \mathrm{~K}, \log g=3.40, \epsilon(\mathrm{He})=0.12$ and $\xi=20 \mathrm{~km} \mathrm{~s}^{-1}$ (dash-dotted). The error bars trace the uncertainty in the rectified flux $\left(f_{\text {rect }}\right)$ due to the $S / N$ of the spectrum $\left( \pm 1 / S / N \cdot 1 / f_{\text {rect }}\right)$.
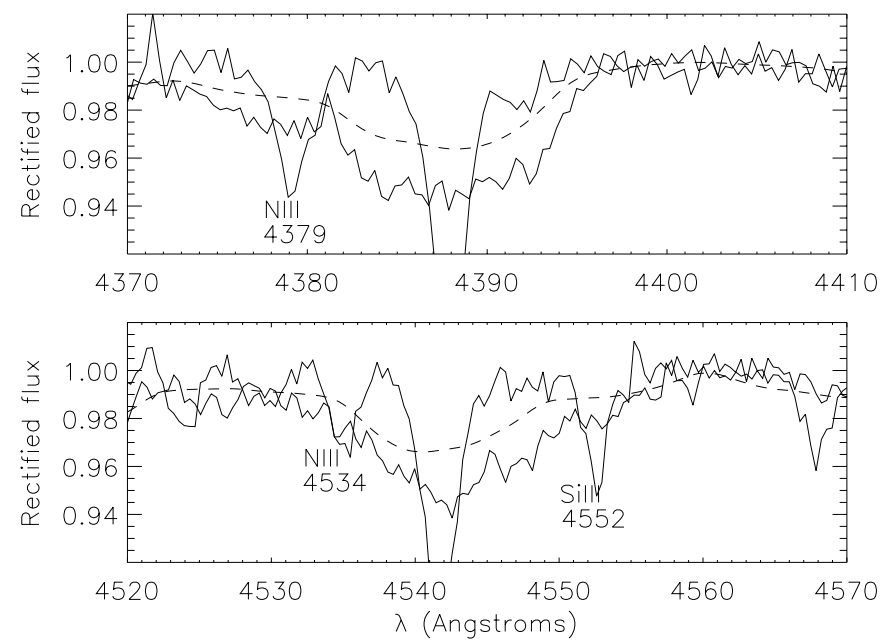

Fig. 6. Line blending in He 4387 and He II 4541. The spectra of HD 209975 (solid line, sharp lines), HD 209975 degraded to $450 \mathrm{~km} \mathrm{~s}^{-1}$ (dotted line) and HD 191423 (solid line, wide features) are shown.
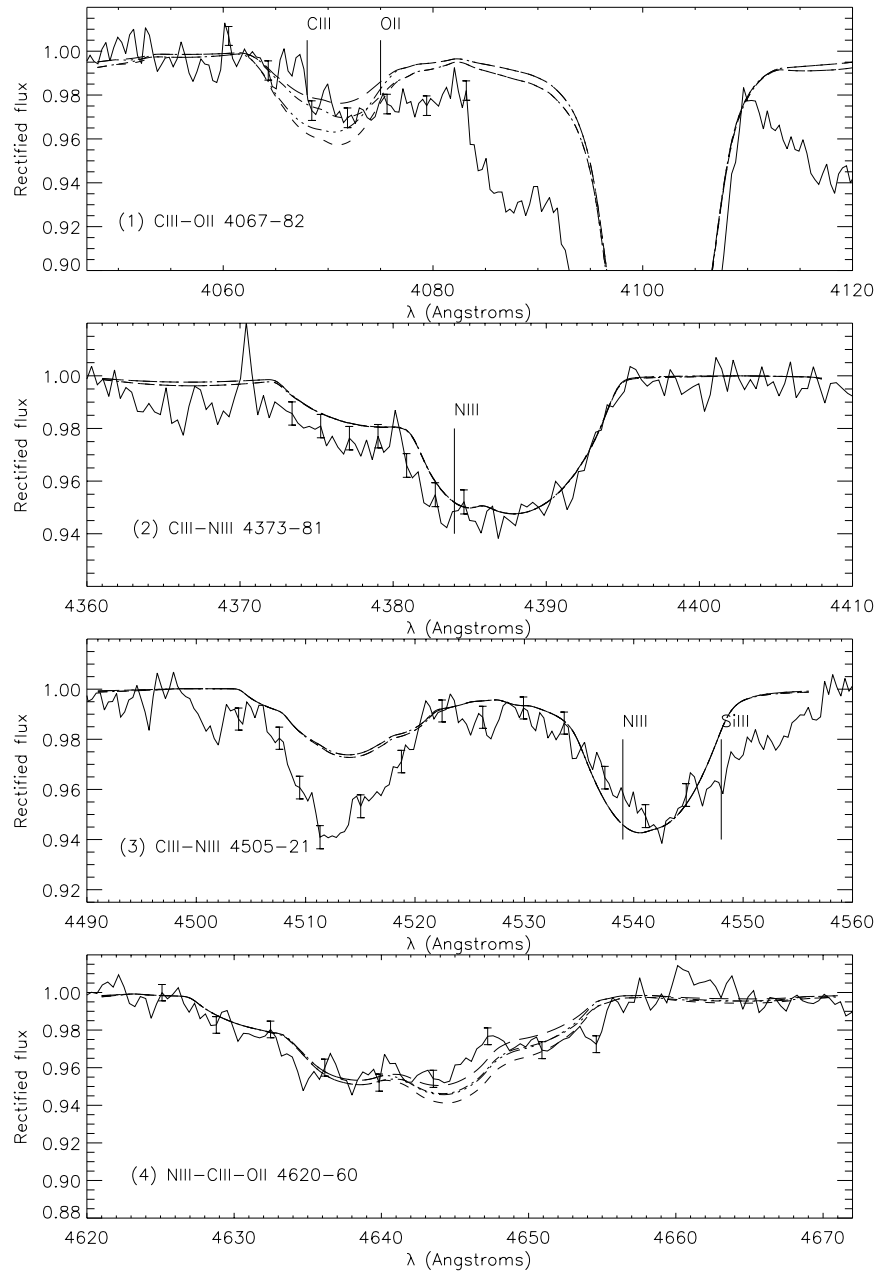

Fig. 7. Spectral synthesis for HD 191423, for the model abundances M0 (short dashed), M1 (dash-dotted), M2 (dash-threedotted), and M3 (long dashed), see text. M1 gives the best fit to blend (1), with $\epsilon(\mathrm{C})=\frac{\epsilon(\mathrm{C})_{\odot}}{10}, \epsilon(\mathrm{O})=\frac{\epsilon(\mathrm{O})_{\odot}}{5}, \epsilon(\mathrm{He})=0.12$ and $\xi=20 \mathrm{~km} \mathrm{~s}^{-1}$.

the abundances of HD 209975 we compute the first model spectrum (model M0, see Fig. 7, short-dashed line).

The blend labeled as (1) is the only one that gives us information about the abundances of $\mathrm{O}$ and $\mathrm{C}$. To find a good fit, starting from M0, we must lower those abundances, which we do in models M1 to M3:

M0: $\epsilon(\mathrm{C})=\frac{\epsilon(\mathrm{C})_{\odot}}{5}, \epsilon(\mathrm{N})=\epsilon(\mathrm{N})_{\odot}, \epsilon(\mathrm{O})=\frac{\epsilon(\mathrm{O})_{\odot}}{5}$
M1: $\epsilon(\mathrm{C})=\frac{\epsilon(\mathrm{C})_{\odot}}{10}, \epsilon(\mathrm{N})=\epsilon(\mathrm{N})_{\odot}, \epsilon(\mathrm{O})=\frac{\epsilon(\mathrm{O})_{\odot}}{5}$
M2: $\epsilon(\mathrm{C})=\frac{\epsilon(\mathrm{C})_{\odot}}{5}, \epsilon(\mathrm{N})=\epsilon(\mathrm{N})_{\odot}, \epsilon(\mathrm{O})=\frac{\epsilon(\mathrm{O})_{\odot}}{10}$
M3: $\epsilon(\mathrm{C})=\frac{\epsilon(\mathrm{C})_{\odot}}{10}, \epsilon(\mathrm{N})=\epsilon(\mathrm{N})_{\odot}, \epsilon(\mathrm{O})=\frac{\epsilon(\mathrm{O})_{\odot}}{10}$.

Blends (2) and (3) have O II components, but as can be seen in Fig. 7, they are dominated by the N III component, the only line that remains at a fixed abundance in models M0 to M3.

Looking at the fits in Fig. 7 we obtain the $\mathrm{C}$ and $\mathrm{O}$ abundances of HD 191423: $\epsilon(\mathrm{C})=\frac{\epsilon(\mathrm{C})_{\odot}}{10}, \epsilon(\mathrm{O})=\frac{\epsilon(\mathrm{O})_{\odot}}{5}$ 

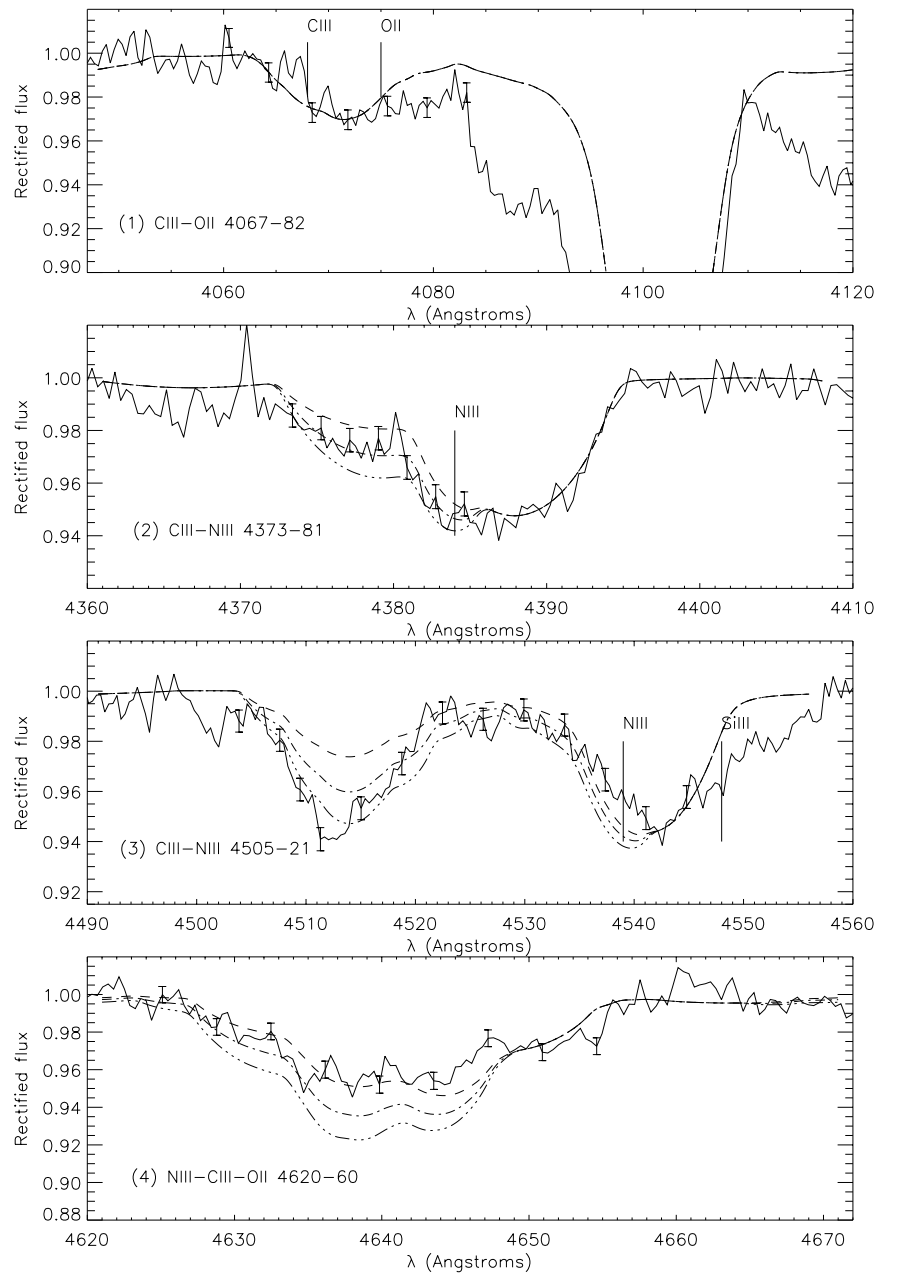

Fig. 8. Spectral synthesis for HD 191423, for the model abundances M1 (dashed), M4 (dash-dotted) and M5 (dash-threedotted) which only differ in the $\mathrm{N}$ abundance. See how blend (2) and (3) indicate different $\mathrm{N}$ abundances (see text).

(model M1, dash-dotted), which are held fixed while we search for the $\mathrm{N}$ abundance, with models M4 and M5:

$$
\begin{aligned}
& \text { M4: } \epsilon(\mathrm{C})=\frac{\epsilon(\mathrm{C})_{\odot}}{10}, \epsilon(\mathrm{N})=2.5 \cdot \epsilon(\mathrm{N})_{\odot}, \epsilon(\mathrm{O})=\frac{\epsilon(\mathrm{O})_{\odot}}{5} \\
& \text { M5: } \epsilon(\mathrm{C})=\frac{\epsilon(\mathrm{C})_{\odot}}{10}, \epsilon(\mathrm{N})=5 \cdot \epsilon(\mathrm{N})_{\odot}, \epsilon(\mathrm{O})=\frac{\epsilon(\mathrm{O})_{\odot}}{5} .
\end{aligned}
$$

In Fig. 8 we see that it is not possible to find a good fit for both blends (2) and (3) with a single value of the $\mathrm{N}$ abundance. While blend (2) and the N III line embedded in He I 4387 are well reproduced for $\epsilon(\mathrm{N})=2.5 \cdot \epsilon(\mathrm{N})_{\odot}$, blend (3) is better reproduced by a higher abundance, $\epsilon(\mathrm{N})=5 \cdot \epsilon(\mathrm{N})_{\odot} \cdot$ Furthermore, the N III line embedded in He II 4541 also gives contradictory information, because it is too strong with the three $\mathrm{N}$ abundances considered. We then find that the $\mathrm{N}$ abundance of this star may be between 2.5 and $5 \epsilon(\mathrm{N})_{\odot}$.

Blend (4) shows evidence of wind emission, as with He II 4686.

Adopting the abundance uncertainties of HD 209975 we give the absolute $\mathrm{CNO}$ abundances of the fast rotator HD 191423:

$$
\log \epsilon(\mathrm{C})=-4.48 \pm 0.24
$$

$\log \epsilon(\mathrm{N}) \in[-3.70,-3.40] \pm 0.17$

$\log \epsilon(\mathrm{O})=-3.82 \pm 0.48$.

From now on we consider the most conservative value for the $\mathrm{N}$ abundance, the lower value, to be the $\mathrm{N}$ abundance of HD 191423.

\section{Discussion}

In Fig. 9 and in Table 13 we compare our results with those of Gies \& Lambert (1992) and Schönberner et al. (1988) for HD 214680, with Gies \& Lambert (1992) for HD 34078, and with Cunha \& Lambert (1994) and Daflon et al. (1999) for the associations Ori OB1 and Cep OB2 respectively. In Cunha \& Lambert $(1992,1994)$ it is shown how the two youngest subgroups of Ori OB1 (Ic and Id) are enriched in $\mathrm{O}$ and $\mathrm{Si}$ with respect to the other two older subgroups (Ia and $\mathrm{Ib}$ ). We compare our results with the mean abundances of the older, non-enriched stars. Also the solar abundances of Grevesse et al. (1996) are quoted.

Due to the strong dependence of the abundance determinations on the particular methodologies followed (see our discussion on this in Sects. 3 and 6), the direct comparison of absolute abundances obtained in different works must be done with care, considering a possible "methodological bias".

For the two dwarfs we see that our results are more consistent with each other than those of Gies \& Lambert (1992), which show considerable differences in the $\mathrm{N}$ and $\mathrm{O}$ abundances of these two similar stars (see Fig. 9, crosses). They do not determine the $\mathrm{C}$ abundances. Schönberner et al. (1988) find CNO abundances of HD 214680 to be higher than ours by 0.3 dex (see Fig. 9, plus signs), an effect that can be explained by the ODFs used in our work, which as seen in Sect. 3.1, lead to lower abundances.

The comparison with Cunha \& Lambert (1994) and Daflon et al. (1999) shows good agreement considering the uncertainties, and it is remarkable that in both their works and our dwarf stars, the $\mathrm{C}$ and $\mathrm{N}$ abundances are more dispersed than the $\mathrm{O}$ abundance, which is practically the same in all cases.

The CNO abundances that we obtain for our two reference dwarf stars are lower than the solar values by $0.1-$ $0.4 \mathrm{dex}$, also in good agreement with the abundances of other unmixed early B dwarfs in the solar neighbourhood (Kilian 1992, 1994; Vrancken 1997). The results therefore support the hypothesis that the Sun is enriched in these elements relative to its neighbour early type stars.

The CNO abundances of our supergiant HD 209975 are different from those of the two reference dwarfs by $\sim 0.1$ dex in the direction of CNO contamination: lower $\mathrm{C}$ and $\mathrm{O}$ and higher $\mathrm{N}$ abundances. However, these small differences are within our uncertainties (also in the relative values, see Table 6 ), so we believe that the differences can be due to the different physical conditions of the star relative to the dwarfs, as we indicated in Sect. 6, and not to real different chemical compositions (remember that it also formed in the solar neighbourhood). 
Table 13. Absolute CNO abundances of our four O9 stars compared to the values in the literature of Gies \& Lambert (1992), Schönberner et al. (1988), Cunha \& Lambert (1994) and Daflon et al. (1999). For HD 191423 we give the abundances corrected for the Galactic gradients of Rolleston et al. (2000) and Gummersbach et al. (1998), see text.

\begin{tabular}{lcccc}
\hline \hline & $\log \epsilon(\mathrm{C})$ & $\log \epsilon(\mathrm{N})$ & $\log \epsilon(\mathrm{O})$ & Ref. \\
\hline HD 214680 & $-3.89 \pm 0.22$ & $-4.19 \pm 0.28$ & $-3.35 \pm 0.27$ & This work \\
O9 V & & $-4.16 \pm 0.31$ & $-3.07 \pm 0.29$ & G\&L \\
& $-3.60 \pm 0.20$ & $-3.92 \pm 0.20$ & $-3.00 \pm 0.20$ & Schön. \\
HD 34078 & $-3.81 \pm 0.16$ & $-4.10 \pm 0.28$ & $-3.36 \pm 0.29$ & This work \\
O9.5 V & & $-4.63 \pm 0.09$ & $-3.55 \pm 0.25$ & G\&L \\
Early B V stars & $-3.65 \pm 0.11$ & $-4.25 \pm 0.11$ & $-3.40 \pm 0.07$ & C\&L \\
in Ori OB1 & & & & \\
HD 209975 & $-3.98 \pm 0.24$ & $-4.01 \pm 0.17$ & $-3.46 \pm 0.48$ & This work \\
O9.5 Ib & & & & \\
Early B V stars & $-3.87 \pm 0.09$ & $-4.42 \pm 0.10$ & $-3.43 \pm 0.10$ & Daflon \\
in Cep OB2 & & & & \\
HD 191423 & $-4.48 \pm 0.24$ & $-3.70 \pm 0.17$ & $-3.82 \pm 0.48$ & This work \\
O9 III:n & $-4.50 \pm 0.24$ & $-3.76 \pm 0.17$ & $-3.87 \pm 0.48$ & Gumm. \\
& $-4.53 \pm 0.24$ & $-3.76 \pm 0.17$ & $-3.87 \pm 0.48$ & Roll. \\
Solar abundances & -3.49 & -4.07 & -3.17 & Grevesse \\
\hline
\end{tabular}

This star gives the reference $\mathrm{CNO}$ abundances of unmixed O9 giants and supergiants, but we will have to study more low gravity stars in order to establish these reference abundances reliably.

The CNO absolute abundances of our fast rotator HD 191423 could be showing the effect of possible internal mixing together with the different original composition of the cloud from which it was formed. It belongs to the Cyg OB8 association, that according to Melnick \& Efremov (1995) forms a group with the associations Cyg OB1 and Cyg OB9. Adopting for our star the galactocentric distance of Cyg OB1 $\left(R_{\mathrm{GC}}=7.8 \mathrm{kpc}\right.$, Massey et al. 1995), we see that it is closer to the galactic center than the Sun, at $8.5 \mathrm{kpc}$ (Gummersbach et al. 1998).

Considering the Galactic abundance gradients found by several authors such as Smartt \& Rolleston (1997b), Gummersbach et al. (1998) and Rolleston et al. (2000), we must correct for the different initial compositions before comparing its abundances with those of our reference stars.

We do so with the most recent gradients of Rolleston et al. (2000):

$$
\begin{aligned}
& \Delta \mathrm{C}=-0.07 \mathrm{dex} / \mathrm{kpc} \times(7.8-8.5) \mathrm{kpc}=+0.05 \mathrm{dex} \\
& \Delta \mathrm{N}=-0.09 \mathrm{dex} / \mathrm{kpc} \times 0.7 \mathrm{kpc}=+0.06 \mathrm{dex} \\
& \Delta \mathrm{O}=-0.067 \mathrm{dex} / \mathrm{kpc} \times 0.7 \mathrm{kpc}=+0.05 \mathrm{dex}
\end{aligned}
$$

that yield the second set of abundances represented in Fig. 9. We see that the corrections are small and tend to reduce the $\mathrm{N}$ overabundance and to increase the $\mathrm{C}$ and $\mathrm{O}$ underabundances. Anyway the trend obtained in the CNO abundances of this fast rotator is in the direction of $\mathrm{CNO}$ contamination.

Massive stellar evolution with rotation predicts that the mixing is more efficient for higher mass stars and for higher initial rotational velocities, with the surface CNO contamination growing as the star ages. Another prediction is that the rotation of the star decreases with time, due to the loss of angular momentum in the stellar wind (Meynet \& Maeder 2000; Heger \& Langer 2000).

The actual spectroscopic masses of our sample stars are the following:

HD 214680, $18 M_{\odot}$

HD 34078, $21 M_{\odot}$

HD 209975, $20 M_{\odot}$

HD 191423, $29 M_{\odot}$

and in Fig. 10 we can see in the HR diagram that our two dwarf stars are certainly young, while the supergiant HD 209975 and the fast rotator are more evolved.

Therefore, the surface CNO contamination found in HD 191423 is in agreement with the evolutionary predictions: the star started its main sequence lifetime with a very high initial rotational velocity and it is now showing the corresponding surface CNO contamination.

In order to obtain more quantitative conclusions we compare our $\mathrm{N} / \mathrm{C}$ and $\mathrm{N} / \mathrm{O}$ ratios with the evolution of these quantities for certain evolutionary models, in Figs. 11 and 12 (Meynet 2001, private communication).

We refer the ratios to their initial values, that for the evolutionary models is the solar composition and for our sample stars are those of the reference He normal stars.

Although we consider the $\mathrm{CNO}$ abundances of HD 209975 as the reference unmixed values for low gravity stars, we show in Figs. 11 and 12 its ratios refered to the reference dwarf HD 214680.

They are compatible with the evolution of a rotating star with initial mass around $20 M_{\odot}$, that has evolved until its actual (projected) rotational velocity. Therefore, we leave open the question of the possible CNO contamination of this object. 

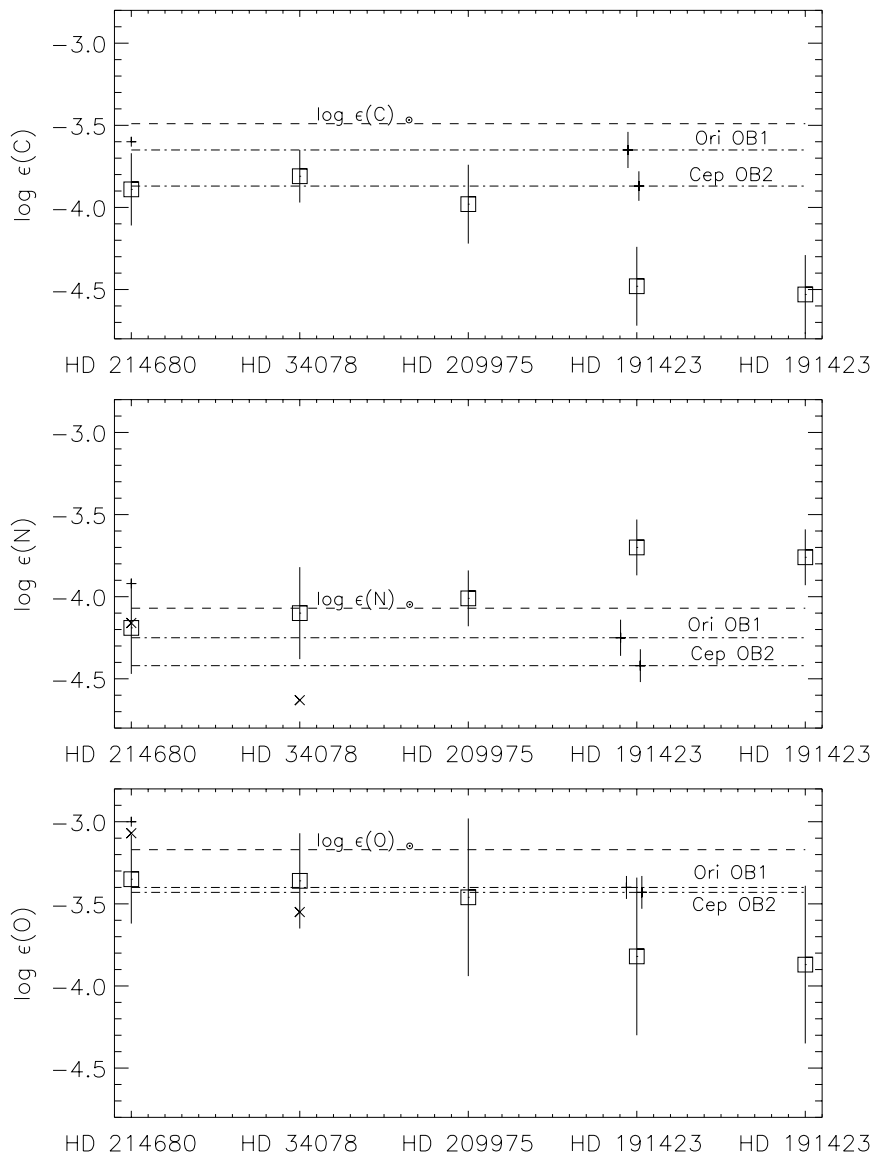

Fig. 9. CNO abundances of our sample stars compared to the values of Gies \& Lambert (1992, crosses) for HD 214680 and HD 34078, and of Schönberner et al. (1988, plus signs) for HD 214680. Also the mean abundances for early B dwarfs in Ori OB1 (Cunha \& Lambert 1994) and in Cep OB2 (Daflon et al. 1999) are plotted (dash-dotted lines with their error bars). Solar abundances are also shown for reference. For HD 191423 we give first the absolute abundances and then the ones corrected for Galactic abundance gradients (see text).

For HD 191423 we can only say that the star must have started its main sequence lifetime with an extremely high rotational velocity, that leads to the present day projected value and surface CNO contamination.

Therefore we find that it is possible to explain our results in the frame of massive stellar evolution with rotation, but we are still far from obtaining quantitative conclusions, due basically to the problem of the projection of the measurable rotational velocities and to the small sample considered.

\section{Conclusions}

We have studied a sample of four Galactic O9 stars in order to investigate the relationship between stellar rotation and surface CNO contamination.

The reference CNO abundances for unmixed O9 stars are given by the three He normal stars studied, all located in the solar neighbourhood. Their chemical compositions are indistinguishable within our uncertainties.

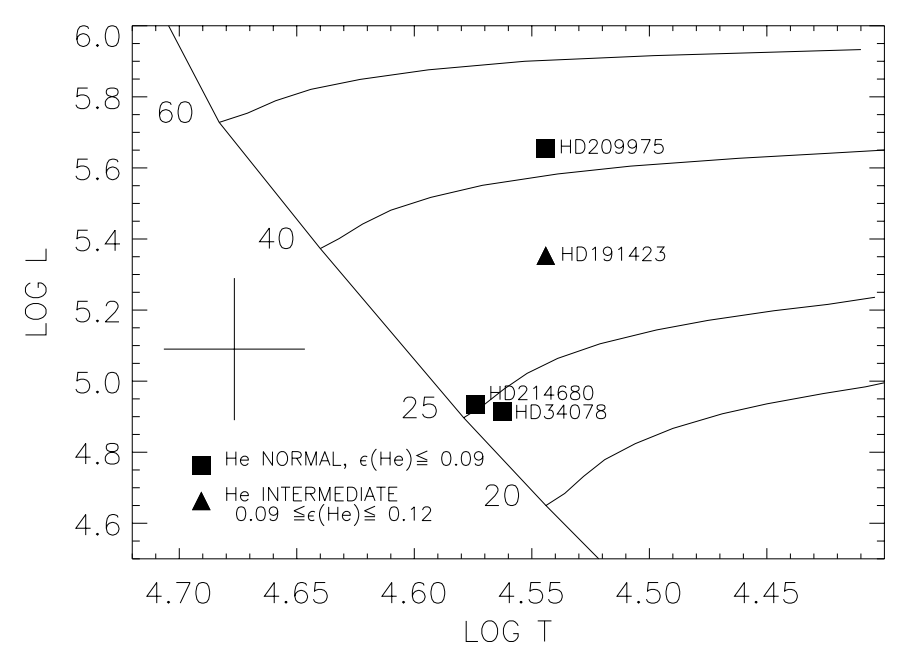

Fig. 10. HR diagram for the O9 stars studied. Classical main sequence evolutionary tracks of Schaller et al. (1992) are shown for the quoted initial masses.

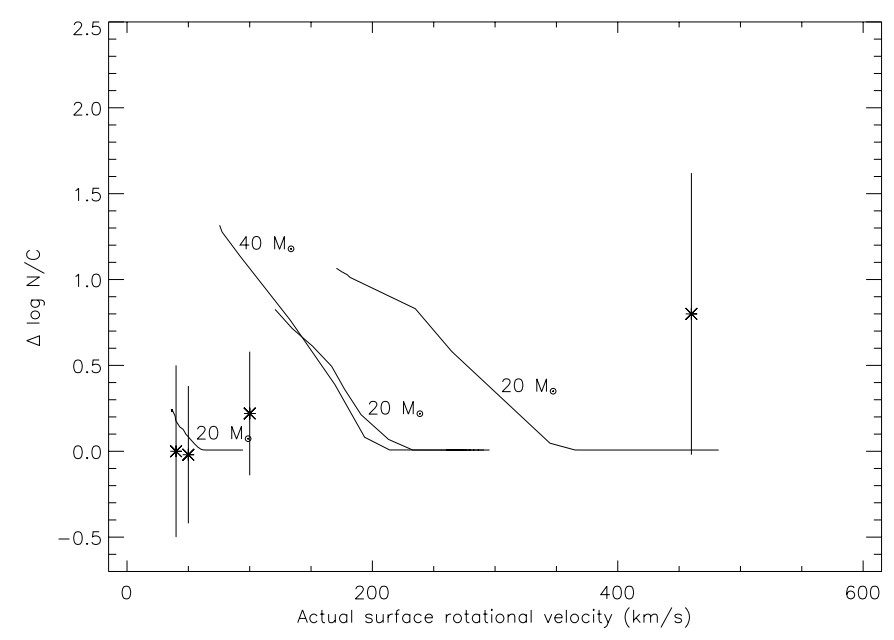

Fig. 11. Evolution of the N/C ratio during the main sequence as a function of the equatorial surface rotational velocity, for models of the quoted initial masses. The observed data are for the projected rotational velocities, and therefore they could all be right shifted to the non-projected values. Uncertainties in $v \sin i$ range from 10 to $20 \mathrm{~km} \mathrm{~s}^{-1}$.

These abundances are in agreement with those found in the solar neighbourhood for unmixed early B stars, with values about 0.2 dex below solar.

The He and CNO abundances of the fast rotator HD 191423 show the trend expected from CNO contamination: $\mathrm{He}$ and $\mathrm{N}$ overabundance and $\mathrm{C}$ and $\mathrm{O}$ depletions. For the reference supergiant HD 209975 we question whether it shows also some surface contamination.

The comparison of our observed $\mathrm{N} / \mathrm{C}$ and $\mathrm{N} / \mathrm{O}$ ratios with the predictions of the evolutionary models with rotation of Meynet \& Maeder (2000) show a qualitative agreement.

Nevertheless, we must be aware of the limitations of this result, which represents only a first step in the investigation of the relationship between stellar rotation and surface CNO contamination in massive O stars. 


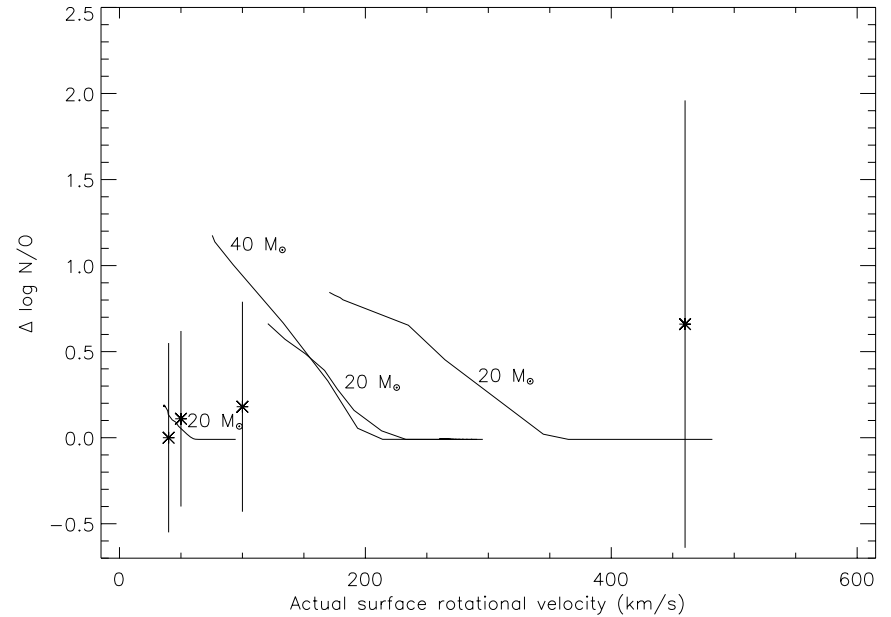

Fig. 12. As Fig. 11 for the $\mathrm{N} / \mathrm{O}$ ratio.

Acknowledgements. We wish to thank N. Przybilla, R. J. García López, G. Meynet and N. Langer for providing us with data necessary for this work and for many valuable suggestions and discussions. AH acknowledges support for this work by the spanish DGES under project PB97-1438-C02-01 and by the Gobierno Autónomo de Canarias under project PI1999/008.

\section{References}

Becker, S. R., \& Butler, K. 1988, A\&A, 201, 232

Becker, S. R., \& Butler, K. 1989, A\&A, 209, 244

Butler, K. 1984, Ph.D. Thesis, University College London

Chiosi, C. 1998, in Stellar Astrophysics for the Local Group, ed. A. Aparicio, A. Herrero, \& F. Sánchez (Cambridge Contemporary Astrophysics), 3

Cowley, C. R. 1971, Observatory, 91, 139

Cunha, K., \& Lambert, D. L. 1992, ApJ, 399, 586

Cunha, K., \& Lambert, D. L. 1994, ApJ, 426, 170

Cunha, K., Smith, V. V., \& Lambert, D. L. 1998, ApJ, 493, 195

Cunto, W., Mendoza, C., Ochsenbein, F., et al. 1993, A\&A, 275, L5

Daflon, S., Cunha, K., \& Becker, S. R. 1999, ApJ, 522, 950

de Zeeuw, P. T., Hoogerwerf, R., de Bruijne, J. H. J., et al. 1999, AJ, 117, 354

Eber, F. 1987, Diplomarbeit, University of Munich

García-López, R. J., Rebolo, R., \& Pérez de Taoro, M. R. 1995, A\&A, 302, 184

Garmany, C. D., \& Stencel, R. E. 1992, A\&AS, 94, 211

Gies, D. R., \& Bolton, C. T. 1986, ApJS, 61, 419

Gies, D. R., \& Lambert, D. L. 1992, ApJ, 387,673

Gold, M. 1984, Diplomarbeit, Ludwig Maximilian Universität

Grevesse, N., Noels, A., \& Sauval, A. J. 1996, in Cosmic abundances, ed. S. S. Holt, \& G. Sonneborn, ASPA, San Francisco, 117

Gummersbach, C. A., Kaufer, A., Schäfer, D. R., et al. 1998, A\&A, 338, 881

Heger, A. 1998, Ph.D. Thesis, Technische Universität München

Heger, A., \& Langer, N. 2000, ApJ, 544, 1016

Herrero, A., Kudritzki, R. P., Vilchez, J. M., et al. 1992, A\&A, 261,209

Herrero, A. 1994, Space Sci. Rev., 66, 137

Herrero, A., Kudritzki, R. P., Gabler, R., et al. 1995, A\&A, 297,556
Herrero, A., Puls, J., \& Villamariz, M. R. 2000, A\&A, 354, 193

Humphreys, R. M. 1978, ApJS, 38, 309

Israelian, G., García-López, R. J., \& Rebolo, R. 1998, ApJ, 507,805

Kane, L., McKeith, C. D., \& Dufton, P. L. 1980, A\&A, 84, 115

Kilian, J. 1992, A\&A, 262, 171

Kilian, J., Montenbruck, O., \& Nissen, P. E. 1994, A\&A, 284, 437

Kudritzki, R. P. 1990, ARA\&A, 28, 303

Kudritzki, R. P. 1992, A\&A, 266, 395

Kupka, F., Piskunov, N. E., Ryabchikova, T. A., et al. 1999, A\&AS, 138, 119 (VALD-2)

Kurucz, R. L. 1979, ApJS, 40, 1

Kurucz, R. L. 1996, in M.A.S.S: Model atmospheres and spectrum synthesis, ed. S. J. Adelman, F. Kupka \& W. W. Weiss, ASP Conf. Ser., 108

Lamers, H. J. G. L. M., \& Achmad, L. 1994, A\&A, 291, 856

Lennon, D. J., Dufton, P. L., \& Fitzsimmons, A. 1993, A\&AS, 97,559

Maeder, A., \& Meynet, G. 2000, ARA\&A, 38

Mason, B. D., Gies, D. R., Hartkopf, W. I., et al. 1998, AJ, 115,821

Massey, P., Johnson, K. E., \& de Gioia-Eastwood, K. 1995, ApJ, 454, 151

Mel'nick, A. M., \& Efremov, Yu. N. 1995, Astron. Lett., 21, 10

McErlean, N. D., Lennon, D. J., \& Dufton, P. L. 1998, A\&A, 329,613

McErlean, N. D., Lennon, D. J., \& Dufton, P. L. 1999, A\&A, 349,553

Meynet, G., \& Maeder, A. 2000, A\&A, 361, 101

Monteverde, M. I., Herrero, A., \& Lennon, D. J. 2000, ApJ, 545,813

Pauldrach, A. W. A, Kudritzki, R. P., Puls, J., et al. 1994, A\&A, 283, 525

Rolleston, W. R. J., Smartt, S. J., Dufton, P. L., \& Ryans, R. S. I. 2000, A\&A, 363, 537

Ryabchikova, T. A., Piskunov, N. E., Stempels, H. C., et al. 1999, Proc. of the 6th International Colloquium on Atomic Spectra and Oscillator Strengths, Victoria BC, Canada 1998, Phys. Scr. T83, 162 (VALD-2)

Santolaya-Rey, A. E., Puls, J., \& Herrero, A. 1997, A\&A, 323, 488

Schaller, G., Schaerer, D., Meynet, G., et al. 1992, A\&AS, 96, 269

Schönberner, D., Herrero, A., Becker, S., et al. 1988, A\&A, 197, 209

Smartt, S. J., Dufton, P. L., \& Rolleston, W. R. J. 1996, A\&A, 310,123

Smartt, S. J., Dufton, P. L., \& Lennon, D. J. 1997a, A\&A, 326, 763

Smartt, S. J., \& Rolleston, W. R. J. 1997b, ApJ, 481, L47

Smith, K. C., \& Howarth, I. D. 1998, MNRAS, 299,1146

Striganov, A. R., \& Sventitskii, N. S. 1968, in Tables of spectral lines of neutral and ionized atoms, IFI/Plenum

Taresch, G., Kudritzki, R. P., Hurwitz, M., et al. 1997, A\&A, 321,531

Venn, K. A. 1995, ApJS, 99, 659

Villamariz, M. R., \& Herrero, A. 2000, A\&A, 357, 597

Villamariz, M. R. 2001, Ph.D. Thesis, La Laguna University

Voels, S. A., Bohannan, B., Abbott, D. C., \& Hummer, D. G. 1989, ApJ, 340, 1073

Vrancken, M. 1997a, Ph.D. Thesis, Vrije Universiteit Brussel

Vrancken, M., Lennon, D. J., Dufton, P. L., \& Lambert, D. L. 2000, A\&A, 358, 639

Walborn, N. 1971, ApJS, 23, 257 